\title{
Integrated Simulation and Modeling Capability for Alternate Magnetic Fusion Concepts
}

\author{
B.I. Cohen \\ E.B. Hooper \\ L.D. Pearlstein \\ L.L. LoDestro \\ J.S. Sarff \\ S.C. Prager \\ T.R. Jarboe
}

November 3, 1998

This is an informal report intended primarily for internal or limited external distribution. The opinions and conclusions stated are those of the author and may or may not be those of the Laboratory.

Work performed under the auspices of the U.S. Department of Energy by the Lawrence Livermore National Laboratory under Contract W-7405-ENG-48. 


\section{DISCLAIMER}

This document was prepared as an account of work sponsored by an agency of the United States Government. Neither the United States Government nor the University of California nor any of their employees, makes any warranty, express or implied, or assumes any legal liability or responsibility for the accuracy, completeness, or usefulness of any information, apparatus, product, or process disclosed, or represents that its use would not infringe privately owned rights. Reference herein to any specific commercial product, process, or service by trade name, trademark, manufacturer, or otherwise, does not necessarily constitute or imply its endorsement, recommendation, or favoring by the United States Government or the University of California. The views and opinions of authors expressed herein do not necessarily state or reflect those of the United States Government or the University of California, and shall not be used for advertising or product endorsement purposes.

This report has been reproduced directly from the best available copy.

Available to DOE and DOE contractors from the

Office of Scientific and Technical Information

P.O. Box 62, Oak Ridge, TN 37831

Prices available from (615) 576 8401, FTS 626-8401

Available to the public from the

National Technical Information Service

U.S. Department of Commerce 5285 Port Royal Rd., Springfield, VA 22161 


\title{
Integrated Simulation and Modeling Capability for Alternate Magnetic Fusion Concepts
}

B.I. Cohen, E.B. Hooper, L.D. Pearlstein, and L.L. LoDestro Magnetic Fusion Energy Division, Lawrence Livermore National Laboratory, University of California Livermore, California 94550

\author{
J.S. Sarff and S.C. Prager \\ Physics Department, University of Wisconsin \\ Madison, Wisconsin 53706
}

T.R. Jarboe

Department of Aeronautical Engneering, University of Washington

Seattle, Washington 98155

\begin{abstract}
This document summarizes a strategic study addressing the development of a comprehensive modeling and simulation capability for magnetic fusion experiments with particular emphasis on devices that are alternatives to the mainline tokamak device. A code development project in this area supports two defined strategic thrust areas in the Magnetic Fusion Energy Program: (1) comprehensive simulation and modeling of magnetic fusion experiments and (2) development, operation, and modeling of magnetic fusion alternateconcept experiments.
\end{abstract}




\section{Outline}

1. Introduction - Numerical modeling of magnetic fusion experiments

2. A comprehensive magnetic fusion modeling code

- CORSICA is a reasonable prototype

- Basics: equilibrium, transport, linear stability analysis, interactive user interface

- Advanced capabilities: CORSICA 2 and CORSICA 3, synthetic diagnotics

3. Development of new prototypes in CORSICA for modeling of alternate confinement approaches.

- Toroidal $\rightarrow$ poloidal flux formulation

- Halo model for plasma outside of the last flux surface

- New diagnostics for CORSICA 1

- Further elaboration of reflectometry simulation, CORSICA 2 and 3

4. Comprehensive modeling of alternate magnetic confinement approaches

- Applications of CORSICA to the Sustained Spheromak Physics Expt.

- Previous computational studies of spheromaks and related plasmas

- Advanced applications of CORSICA to spheromaks

- Proposed applications of CORSICA to reversed field pinches (RFPs)

5. Program directions

- National Transport Code initiative

- DOE ER strategic simulation projects (SSP)

- New collaborations to support spheromak and RFP research, e.g., LLNLUWash-UWisc., LLNL-General Atomics-UWisc. 


\section{Introduction - Numerical Modeling of Magnetic Fusion Experiments}

Both magnetic fusion and inertial confinement fusion programs around the world are very dependent on comprehensive numerical modeling. This applies to the analysis of physics concepts, the design and operation of experiments, and the analysis of experimental data. The outstanding example of a comprehensive fusion modeling code is LASNEX ${ }^{1}$ developed at LLNL which is used in the inertial confinement fusion program. LASNEX combines multiple physics packages that allow the user to follow the temporal evolution of the relevant phenomena in two spatial dimensions and postprocess the simulation to extract various diagnostics taking advantage of a flexible interactive user interface provided by BASIS 2 also developed at LLNL that enables diverse new applications to be launched during an interaction session without recompiling the code. LASNEX has been tremendously successful in integrating the physics of inertial fusion into a flexible and powerful modeling tool used by a large user community. LASNEX has had a large impact on the inertial fusion theory and experimental programs.

The U.S. magnetic fusion program relies heavily on a suite of physics codes to help with the design, operation, and analysis of experiments and to assist theory. Important examples are the magnetohydrodynamic equilibrium and stability codes and the transport codes, i.e., hydrodynamic codes (typically in one spatial dimension) that solve plasma fluid equations as functions of time following the conservation of mass, momentum, and energy with sources and sinks to model experiments. The solution of the magnetohydrodynamic equilibrium equation reduced to the Grad-Shafranov equation detemines the self-consistent relation between the magnetic flux and the plasma pressure and currents. ${ }^{3}$ Examples of the important magnetic fusion transport codes are TRANSP, WHIST, ONETWO, BALDUR, TSC, and CORSICA 1. These codes support the major U.S. tokamak experiments and the ITER project. These codes are generally specific to the tokamak configuration. The tokamak transport codes are relatively mature and very successful in supporting the tokamak theory and experimental programs.

The magnetic fusion program has long supported a research program investigating a variety of confinement approaches. The mainline of research has focused on the tokamak, but alternate concept confinement approaches continue to receive attention, and the Office of Fusion Energy Sciences has a renewed commitment to support a vigorous research program in alternate approaches. Examples of alternate approaches are the straight and toroidal $\mathrm{Z}$ pinches, the $\theta$ pinch, reversed-field pinch (RFP), mirror and tandem mirror, field-reversed configuration (FRC), field-reversed mirror, spherical tokamaks, spheromaks, torsatrons, and others (Figure 1). Fusion researchers continue to explore alternate confinement approaches both theoretically and 
experimentally in order to better understand the plasma physics and to improve the prospects for controlled fusion.

In order to better understand experiments in alternates, to improve their design and performance, and generally to get the most from the resources invested, it is essential to have a high quality modeling capability. A modeling capability for alternate fusion concepts comparable to LASNEX or to that which exists for tokamaks is largely non-existent. Thus, there is a great need for developing a comprehensive modeling capability for alternates and an excellent opportunity to develop a new program in this area.

This study investigates what can be done to develop a new capability for numerically modeling alternate confinement fusion experiments. Because the LLNL Magnetic Fusion Energy Program is currently undertaking a new spheromak experiment, the Sustained Spheromak Physics Experiment (SSPX), we have already begun extending the comprehensive tokamak modeling code CORSICA to address spheromak configurations. This study describes the more general extension of CORSICA to address alternate concepts as well as extensions specific to spheromak modeling.

\section{A Comprehensive Magnetic Fusion Modeling Code}

The comprehensive numerical modeling of a magnetic fusion experiment requires the integration of a number of distinct calculations of physics phenomena that operate on widely different time and space scales and whose coupling to one another may be very strong and direct in some cases and very weak and indirect in others. A brute-force, direct approach to the numerical solution of the primitive equations describing all of the plasma physics, atomic physics and radiation, the physics of neutrals, and the interaction of plasma and neutrals with material walls is hopelessly impractical. Instead, analytical reduction of the primitive equations and simplified models are adopted for specific important physics elements; and other physics elements receive attention separately. The specific physics elements are married together taking advantage of the separation of time and space scales. This will be clarified by taking up the discussion of a specific family of physics packages embodied in the CORSICA tokamak modeling code. ${ }^{4}$ CORSICA is a reasonable prototype for a comprehensive magnetic fusion modeling code (Figure 2); indeed, some CORSICA packages have already been used for the spheromak.

CORSICA was developed at LLNL under LDRD funding as a Strategic Initiative. The Office of Fusion Energy Sciences continues to fund CORSICA applications and additional development. At its heart, CORSICA contains a one-dimensional (with respect to a radial-like spatial variable) transport code (time-dependent numerical solution of particle, momentum, energy, and magnetic flux transport fluid equations) married to two-dimensional 
calculation of the free or fixed-boundary magnetohydrodynamic equilibrium (TEQ) to determine the magnetic geometry for a toroidally symmetric configuration (Figure 3). This captures the most important physics in the tokamak core. The one-dimensional transport model takes advantage of the fast transit of free-streaming plasma along the field lines to render homogeneous the variation of a number of plasma quantities in the magnetic flux surfaces so that only the variations across the flux surfaces are strong enough to be retained in the transport equations on the slow timescale of the cross-field transport. The transport equations are augmented with various models for plasma particle and energy sources and sinks. In some cases there are separate source and sink simulation packages used to calculate a contribution to a transport coefficient or a source/sink term in the transport equations. The combination of computational packages to calculate onedimensional transport and two-dimensional magnetohydrodynamic equilibrium is CORSICA 1 . CORSICA 1 includes the physics needed to determine stability of the equilibrium to vertical displacements, and it also evaluates the Mercier stability criteria for high mode number ballooning stability. CORSICA 1 has been used heavily by the LLNL-General Atomics collaboration to model the DIII-D tokamak and by researchers modeling ITER (Figs. 4-6). 5,6

CORSICA was extended beyond the traditional capabilities of magnetic fusion transport codes to allow it to couple self-consistently to calculations of other important physics elements relevant to describing tokamak experiments. CORSICA 2 couples the two-dimensional time-dependent fluid code UEDGE, ${ }^{7}$ which models the edge plasma, to CORSICA 1, which models the core (Figure 7). ${ }^{8}$ UEDGE retains the assumption of toroidal axisymmetry but uses two spatial variables to capture the physics of the magnetic geometry in the edge plasma near the divertor and a magnetic X-point. An iteration technique is used to achieve continuity of the fluid transport quantities at the core-edge interface. Theory and experiment have established that strong variations of the radial electric field near the tokamak core-edge interface produce strongly sheared flow that shears apart the turbulent eddies produced by drift-wave instabilities and thus reduces the turbulent transport. This in turn improves the energy confinement of the plasma. The coupled core-edge simulations in CORSICA have been used to demonstrate the transition to improved confinement associated with "H-modes" of operation in the major tokamak experiments (Figure 8).

Unless drift-wave or resistive mode driven turbulence is suppressed by a transport barrier, e.g., strongly sheared electric fields producing shear flow, core energy confinement in tokamaks is significantly degraded relative to plasmas with much less drift-wave turbulence. Various reduced models for turbulent drift-wave transport have been coupled to CORSICA. A step toward a model that is closer to first principles has been to couple CORSICA 1 to a three-dimensional, time-dependent fluid transport code (two different 
turbulence codes have been coupled to CORSICA). The turbulence code is coupled to CORSICA 1 by averaging its fluxes, estimating transport coefficients, and passing these to to the CORSICA 1 transport code, which then predicts the plasma profiles and that drive the turbulence. The codes are iterated until the profiles and turbulent fluxes are consistent. Although the turbulence code is required to use a much smaller timestep than does the transport code, the iteration procedure does not require that the coupled system be constrained to use the small timestep of the turbulence calculation for everything. The successfully coupled code is called CORSICA 3.

CORSICA has also been extended to include a few emulated (synthetic) diagnostics. ${ }^{9}$ These diagnostic packages, reflectometry and a soft X-ray diagnostic, simulate the diagnostic instruments on experiments so that a more direct comparison of the CORSICA modeling and the actual experimental data can be made and so that assumptions and inferences made in the data analysis algorithms for the experiments can be tested. A distributed computing package has also been added to CORSICA to provide access to data files stored on the LLNL MFE network. This allows researchers to have access to the DIII-D tokamak database from the CORSICA command line. The reflectometry density and magnetic field diagnostic package uses plasma and magnetic field profiles computed in CORSICA 1 or obtained from the experimental database as input. The electromagnetic wave equation is solved for the one-dimensional propagation of ordinary or extraordinary mode microwaves across the magnetic field lines on a radial chord to their cutoffs as a function of frequency and plasma parameters. The microwaves are reflected back to a detector in the simulation, and postprocessing routines mimic the actual experimental diagnostic. The results are reconstructions of the electron density and magnetic field radial profiles (Figures 9 and 10). Models of the soft $\mathrm{x}$-ray emission and the silicon detector sensitivity are incorporated in the soft $x$-ray diagnostic simulation. The diagnostic provides multiple chord measurements of the volume-averaged emissivity from which the shape of the plasma density and temperature can be inferred (Figure 11).

Figure 2 presents a schematic of the key elements in CORSICA. We consider CORSICA to be a reasonable prototype for a comprehensive tokamak modeling code. An essential ingredient in CORSICA that continues to facilitate integrating its parts, and enables flexible interactive use of the code, rapid prototyping of new calculations, and the invention of new code diagnostics on the fly is the BASIS system developed at LLNL. ${ }^{2}$ BASIS has a built-in parser to provide a flexible user interface, built-in plotting and I/O capability, a restart capability, and a number of high-level mathematical functions. 


\section{Development of New Prototypes in CORSICA for Modeling of Alternate Confinement Approaches}

\section{CORSICA 1}

This reports the progress in the expansion of CORSICA capabilitics to model alternate toroidal configurations. CORSICA 1 solves the Grad-Shafranov equation to specify the plasma equilibrium with the magnetic fields. The Grad-Shafranov equation is driven by two arbitrary functions of either toroidal or poloidal magnetic flux (neglecting rotation), the plasma pressure, $P$, and $F=R B_{\text {tor. }}$ ( $R$ is the major radius and $B_{\text {tor }}$ is the toroidal magnetic field. These two functions of flux are then determined by the transport equations and the Ohm's law equation. Because transport along the ficld lines is much more rapid than transport across field lines, this equation can be averaged over a flux surface generating one-dimensional equations.

The choice of independent coordinate must be a flux function, but otherwise is arbitrary. For tokamaks, where the toroidal field is very large and approximately time independent (the kinetic pressure over the magnetic pressure is small), the standard choice is the toroidal flux. For RFP's this choice is not possible since the toroidal field changes sign and the toroidal flux is not single valued. In this case a proper choice would be the poloidal flux, which is single valued. For a classical spheromak where the toroidal magnetic field goes to zero on the edge, the poloidal flux is again a better choice to avoid diverging terms a the plasma edge. For a driven spheromak, with currents flowing through the gun, the toroidal field is no longer zero on the last closed surface; nonetheless, the poloidal flux is still the better choice. In any event, the toroidal and poloidal fields are now of comparable size; and both will be changing as functions of time. This offers a further complexity, namely an approximately fixed density and temperature in space will be strongly time varying in the Lagrangian frame. This is rather different from the tokamak as indicated above. To properly account for this we must now include the classical pinch and transport terms. We have worked out the implications of these changes and modified CORSICA accordingly. The modification of the code took two man-months. It should be noted that this change now makes CORSICA unique. The fixed-boundary transport codes, such as TRANSP, BALDUR and WHIST, and the free-boundary codes TSC and DINA are still formulated in terms of the toroidal flux.

We have completed a gun model for the driven spheromak. For this application, we presently assume that the plasma outside the last closed flux surface, which connects to the gun, is in the "Taylor relaxed state," for which the magnetic energy is a minimum subject to conservation of magnetic helicity. Helicity is a measure of the linking of toroidal and poloidal fluxes. We have also partially completed a halo model which will relax this constraint for the spheromak and allow us to study the axisymmetric effects 
of tokamak disruptions. To complete this work will require about another man month of effort. The last improvement to CORSIC $\Lambda 1$ is a new diagnostic package which uses the measured tangential magnetic field at prescribed magnetic loops to reconstruct the $\lambda$, i.e., $F^{\prime}$, profile (the ratio of the parallel current to the magnetic field at zero plasma pressure) and the plasma current. This package is incorporated into the code but is not completely debugged.

\section{CORSICA 2: Core/Edge Code Coupling Across an Interface}

The aim of the CORSICA 2 project was to extend the simulation of the tokamak plasma to include an accurate description the relatively cold plasma at the edge (the outermost closed flux surfaces) and beyond, i.e., in the "scrape-off-layer" (SOL). The SOL plasma is primarily terminated by "divertor plates" or other material limiters within a few centimeters in the radial direction of the last closed surface (because the first open field-lines provide particles a direct, rapid parallel path to a material surface), but is also to some extent in contact with the vacuum chamber wall.

Although the edge and SOL carry only a minor fraction of the current and so have little influence on the magnetic equilibrium, and are too cold to contribute appreciable fusion power, understanding of this plasma through experiment and modeling is nevertheless an important part of the MFE program, for two reasons. First, the heat-flux load from the core plasma---a result of the power injected into the core and the core's finite energyconfinement time---on the terminating plate is sufficiently high that it is a challenge to design a plate that will survive reactor conditions. Second, core transport properties are a highly complicated affair; among the most favorable regimes discovered experimentally to date are those which display significant edge-structure and/or sensitivity to conditions in the edge. Thus, although core temperatures are two orders of magnitude higher than the edge's and the heat and particles flow outward, simulation of the tokamak core (which regime or transport "mode" is it in? what are the radial density and temperature profiles, and the global averages?) depends to a surprising degree on the radial boundary condition for the core transport calculation. This boundary condition is of course also needed as input by any model of the SOL and divertor plasma.

Apart from the question of the transport coefficients themselves, which in the SOL must span both long and short mean-free path regimes, modeling transport in the edge and SOL is considerably more complicated than in the core: one must include interactions with neutrals (and calculate the neutralgas profiles), surfaces, and radiation, and take careful account of the various impurity species. But from the point of view of CORSICA, the distinguishing feature of the edge/SOL plasma is that although it is still toroidally symmetric (neglecting fluctuations), it is no longer 1D: the plasma is sufficiently cold that 
the mean-free path shrinks enough that gradients develop parallel to magnetic field lines. For these reasons state-of-the art edge/SOL codes are significantly more expensive per step than $1 \& 1 / 2 \mathrm{D}$ free-boundary core transport codes (and the former do not even, to date, solve for the magnetic geometry). Furthermore, once parallel transport is included it brings in a very short timescale (in some parts of the SOL it remains very fast compared to the perpendicular transport; note there are also some very fast yet nonignorable atomic physics timescales) which now must be simulated; and the perpendicular timescale itself is significantly shorter than core confinement times. Thus, although it would be possible to simulate the entire axisymmetric tokamak with an edge code running over the entire plasma volume and generalized to include the evolving magnetics, we have in CORSICA 2 developed a much more efficient coupled-code approach: the edge/SOL-code volume extends inward only until it reaches the last of the 1D core surfaces, where it shares a 1D boundary condition with the core, which is determined as part of the solution, and --possible with such a coupled scheme-- only the edge code is run with the required short edge timesteps. (Alternately, the edge code is run in steady-state mode; that is, due to its shorter timescales the edge/SOL is modeled on the core transport timescale as always being in equilibrium with the shared radial boundary condition). For each coupled field, the boundary condition is determined by varying the shared value of the field (or flux) at the coupling surface such that both codes return the same flux (or field).

In the course of the CORSICA Director's Initiative, two quite different schemes to implement the coupling were devised and compared, one of which is implemented in the standard version of CORSICA. The edge code employed was LLNL's UEDGE -- the major US tokamak edge and divertor modelling code. Five fields $\left(n_{D}, n_{\alpha}, n_{g a s}, T_{e}\right.$, and $\left.T_{i}\right)$ are included in the coupling. Proof-of-principle coupled simulations were carried out. ${ }^{7}$ At the present time, CORSICA 2 is being revived, extended (to include coupled flowfields), and developed into a practical tool for physics applications, driven by interest from the DIIID experimental program at GA.

Application of CORSICA 2 to axisymmetric alternate-concept devices should be straightforward, assuming the core and edge codes have been adequately adapted. UEDGE is presently being adapted to the spheromak, including a much higher edge current than in the tokamak, plasmas processes near current-emitting surfaces, and other effects. The long-term goal of this development is to use the spheromak UEDGE code in CORSICA 2.

An area in which further attention and possible algorithm development are required, for tokamaks as well as alternates, is the inclusion of diffusion coefficients, in both edge and core, modeling instability-driven turbulent transport, along with a transport equation for the turbulent field energy. Preliminary looks at such models (outside of CORSICA 1 and UEDGE), which 
are stiffly nonlinear and exhibit the bifurcations expected to support the experimentally observed transitions between transport modes, reveal they can easily give rise to differential equations with ill-determined (numerically, if not analytically) solutions.

\section{CORSICA 3: 1D Transport with Turbulence-Driven Fluxes Self-consistently Included}

Microturbulence in tokamaks has long been considered to have a significant impact on the evolution of the averaged fields, and in particular to be the primary mechanism for transport of energy from the interior of the plasma to the edge and thence the surrounding structures. The turbulent fluctuations in the plasma density, temperature, electrostatic potential, etc., are themselves driven by gradients of the averages of these fields. Quantitative modeling of this turbulence-transport system is thus essential to predict the performance of future large machines.

Turbulence simulations must obviously operate on a timescale short enough to capture effects of the highest important frequencies in the problem. If they allow the averaged profiles ---the turbulence drive--- to evolve (via modecoupling to the lowest mode-number), these codes can, if run long enough and with enough other physics added, be used alone to simulate the whole device. However, the time for the averaged profiles to evolve is typically much longer than the time it takes for the turbulence code to reach a saturated steady state with the averaged profiles frozen. Thus the many steps required for the 2 or 3D turbulence code (expensive per step cf. a 1D core transport code) to reach a steady state with fixed profiles would be multipled by a large separation-of-timescale factor were it to be used to simulate plasma evolution on the transport timescale.

The methods of CORSICA 3, predicated on the existence of this separation of timescales, are designed to eliminate the expense associated with it. The basic scheme continues to advance the averaged profiles with 1D transport equations with steps appropriate for simulating the profile evolution (or only one step if the steady state solution is sought); each such transport step is run in concert with a turbulence simulation. During the iterations of the $1 \mathrm{D}$ transport calculation (iterations are always necessary because the 1D equations are nonlinear and an implicit scheme is required for numerical stabilty), the turbulence code is run one or more timesteps (much shorter), the turbulencecode flux is surface averaged and this latest result, divided by minus the latest 1D gradient profile, is used for the anomalous diffusion coefficient in the next iteration of the 1D transport equations. The turbulence code is run in fixedprofile mode; the profiles are not in fact fixed, but they are legislated and reflect the latest iterative solution to the $1 \mathrm{D}$ transport equations. Thus at the end of each transport timestep, no matter what its size, one has obtained a solution including a turbulence- generated flux which is self-consistent with 
the profiles at the end of the transport timestep, i.e., fully implicitly, for a price comparable to a single run of the turbulence to saturation with fixed profiles. (The implictness is important: otherwise the coupled 1D/turbulencecode set would be driven unstable by the short turbulent timescales; note that it has been achieved without computing Jacobians, which would be prohibitively expensive to obtain here, and without indeed knowing anything at all about the functional dependence of the turbulent flux on the profiles.)

A number of issues must be dealt with for practical application of the scheme. First, analysis using model gradient-dependent turbulent fluxes, shows that the iterative scheme just described can be iteratively unstable; this can be cured by averaging over the iterations. Second, there is no guarantee that the computation of the anomalous diffusion coefficient as just described will yield a positive number. Two methods, about equally successful, have been devised to deal with this problem. One can assign a floor to the diffusion coefficient, and add a convective term such that combined terms sum to the correct anomalous flux; or one can work with a changed variable, which does behave diffusively, by adding a dynamically computed but known component (an "adaptive alias") to the field. Third, a global turbulence code may not be available. The state-of-the-art codes which simulate turbulence in tokamaks are very time-consuming to run, even with fixed profiles. Therefore, the fact that there are two spatial scales in the problem (wavelength and gradient scale-length) is often exploited and codes have been developed in the "local" approximation, i.e., with radial gradients of the surface-averaged profiles held constant in radius. (In this case one is guaranteed a positive anomalous diffusion coefficient.) To obtain transport timescale simulations in this case (which impossible to do with a stand-alone local turbulence code), the scheme calls for completely independent turbulence code runs to be launched, with input gradient and other parameters set to their local values across the core radius by transport code.

The first application of the method was a pilot project employing a set of $2 \mathrm{D}$ equations in a slab, derived by Hasegawa and Wakatani for modeling electrostatic drift-wave turbulence in a tokamak edge. Both local and global implementations of the coupling worked well. For problems where there is at least a moderate separation of spatial scales between the fluctuations and the background, the coupled approach achieves significant savings over the comparison stand-alone simulations, whether or not global effects emerge, while finding, to within expected statistical variations, the same averaged profiles.

For the parameters used in our simulations and for parameters of typical interest for drift-wave-type turbulence in tokamaks, the global implementation of the coupling (in which a single large turbulence code with the same radial domain as the transport code is used, as described above in 
outlining the method) would be more efficient than the local one (in which separate copies of the turbulence code are used at each transport mesh-point).

CORSICA 3 itself incorporates the ability to communicate with turbulence simulation codes (via distributed computing, if desired) and the implementation of our turbulence coupling algorithm. Using the premier 3D fluid tokamak core turbulence code, the radially local gyrofluid code GRYFFIN developed at the Princeton Plasma Physics Laboratory by Hammett, Dorland, and Beer, initial experiments were performed coupling CORSICA's transport module to ion-temperature-gradient turbulence simulations. Coupling to a band of eight GRYFFIN's in the outer portion of a tokamak core (which nevertheless encompassed significant variation of parameters), singletimestep steady-state self-consistent converged runs were obtained with a straightforward application of the basic scheme and techniques developed in 2D. Full-core coupled calculations proved much more difficult. The main problem -- which should be kept in mind when contemplating coupling to any code under rapid development and at the edge of computational feasibility -- was that GRYFFIN is not a generally robust code, and was significantly less so in the much more unstable inner core. Also, the flux becomes a very sensitive function of the profiles in this regime. Finally, during the iteration the profiles developed extrema, and thus even the iteration-averaged local gradients computed for the local codes in its vicinity, before it settled down, would pass through very wide parameter swings. A number of controls were developed that monitored many of the coupled variables and attempted to steer the iteration through difficulties, and in the end a converged self-consistent simulation over much, but not all, of the core was obtained.

A number of issues remain before CORSICA 3 techniques will yield coupled turbulence/transport simulations of practical use. For the coupling of multiple fields, off-diagonal contributions of the gradients to the fluxes, which can be significant and of either sign, and whose analytic form in general will not be known, can have a serious impact on the stability of the method. Stability of the coupling algorithm near critical gradients (that is, values of the gradients at which the turbulence-driven diffusion coefficient vanishes) is also a potential issue. We have carried out initial analysis and some testing of various algorithms in this regard, and feel optimistic that adequate techniques can be devised.

--The coupling control algorithm must be made adequately robust (enough so that new cases do not in general require more fixes).

--The transport-equation sources must be included in the iteration scheme, to make them self-consistent with the profiles (so far they are frozen); due to the strong nonlinearities, this will bring in more iterative stability issues. 
--There is the possibility that the transport-inducing turbulence is electromagnetic in origin, e.g., finite-beta resistive ballooning modes. Here one would want to couple to an evolving equilibrium as well, in order to take into account the shear in the magnetic field.

Finally it should be remarked that implementation of both the CORSICA 2 and 3 coupling schemes as well as the algorithm development for CORSICA 3 were enormously facilitated by having the BASIS system available.

\section{Reflectometry Extensions}

In the last year we have added new reflectometry simulation capability to CORSICA and undertaken a series of simulations in support of the ultrashort-pulse reflectometer (USPR) being built for SSPX. We added the capability to solve the full wave equation describing the propagation and linear mode conversion of mixed polarization microwaves (ordinary and extraordianry waves) in one dimension and extended this to two dimensions. From the reflected mode-converted signals as a function of frequency, we have demonstrated that the shear in the magnetic pitch angle along a radial chord in the plasma midplane can be reconstructed (Figure 12). Progress has been made in understanding the signature of magnetic shear perturbations in the linear mode-conversion reflected signals and their effects on the reconstruction of the magnetic pitch angle profile (Figure 13). When combined with the reflectometry data on the group delays for ordinary and extraordinary modes from which the spatial profiles of the electron density and modulus of the magnetic field are deduced, the pitch-angle profile allows one to infer the plasma current profile in the midplane from the curl of the vector magnetic field profile assuming axisymmetry and approximate updown symmetry. ${ }^{10}$ The simulations have revealed much of the underlying physics that will influence the reflectometer for the anticipated SSPX profiles, have led to an understanding of the resolution requirements and signal-tonoise issues in the reconstructions, and produced software that will be used in the profile reconstructions using the experimental data.

\section{Comprehensive Modeling of Alternate Magnetic Confinement Approaches}

\section{Applications of CORSICA to the Sustained Spheromak Physics Experiment}

CORSICA has been used both to model spheromak physics and to design the Sustained Spheromak Physics Experiment, SSPX. The uses of CORSICA have included:

- Analyzing the magnetic field equilibria of spheromaks in the ideal MHD approximation (Figure 14); ${ }^{11}$

- Modeling the coupling between the spheromak and the helicity injector which generates and sustains the spheromak (Figs. 15-17), 12,13 with different 
expressions for the current profiles inside the magnetic separatrix and in the injector and edge plasma regions;

- Determining the stable limits of $\beta$ (ratio of plasma kinetic energy density to magnetic field density); ${ }^{11,12}$

- Evaluating the stability to current-driven modes by providing equilibria to a MHD stability code, "GATO," at General Atomics);14

- Benchmarking a new resistive, three-dimensional MHD code, "NIMROD," being developed by the national Magnetic Fusion Energy Community;

- Studying the effect of enhanced magnetic turbulence and resulting energy transport during the startup phase of SSPX (Figure 18);15

- Modeling the response of an Ultra-Short-Pulse Reflectometer, USPR, being developed in collaboration with U.C. Davis to measure density and magnetic field profiles and fluctuations; 10 and

- Modeling the magnetic configuration of possible spheromak power reactors, including a pulsed, high-beta concept which has the potential of changing the paradigm of magnetic fusion reactor design. ${ }^{16}$

Many of these calculations have been used to optimize the geometric shape and dimensions of SSPX. The coils to generate the bias magnetic field in the helicity injector have been designed using CORSICA to ensure that the vacuum magnetic field in the flux conserver is very small, thus minimizing magnetic field errors which are known to enhance helicity and energy losses. The resulting design is thus much better based on analysis of the known physics than was possible for previous spheromak experiments.

\section{Previous Computational Studies of Spheromaks and Related Plasmas}

CORSICA has computational capabilities similar to those applied to other experiments, but contains a broader range of physics and is more comprehensive in nature. In addition, it provides very fast turn-around time and is easily applied to studies of plasma equilibria, transport, and other problems of interest. Previous experiments have used several codes to analyze the physics; examples are the Compact Torus Experiment, CTX (Los Alamos) and the Helicity Injection Tokamak, HIT (University of Washington):

CTX. In the spheromak CTX, individual codes were used to address some of these same issues. Markin did equilibrium and stability analysis with his own Grad-Shafranov solver and ideal MHD stability analysis code. The equilibrium was calculated in the actual experimental geometry using a 
profile of $\lambda$ linear in normalized poloidal flux, $\psi$, so $\lambda(\psi)=\lambda_{\text {ave }}[1+a(2 \psi-1)]$ where $\alpha$ is the only free parameter, and $\lambda_{a v e}$ is determined by the geometry and $\alpha .{ }^{17}$ In this work $\alpha$ was manually adjusted to give a good fit to the surface magnetic field data. For stability studies, this same $\lambda(\psi)$ was used in a rectangular cross section "tuna can" flux conserver with no open flux. The calculated profiles were observed to be unstable approximately when the stability analysis predicted. However, the $n=1$ mode was calculated to be slightly more stable than observed, probably because the destabilizing entrance cylinder was not present in the calculations. However, the observed pressure gradients in CTX from multi-point Thomson scattering were much higher (factor of 20) then the Mercier limits. The linear $\lambda$ profile is believed to be inadequate for $\beta$ limits which depend strongly on the detailed profile; this dependence has been confirmed using CORSICA which has considerable freedom in profiles. Internal magnetic measurements are needed to do better than a linear $\lambda$ fit to experimental data. The only transport work done on CTX was measuring the global energy confinement during decay. Measuring the magnetic energy decay rate from the magnetics as described above and using multi-point Thomson scattering for electron temperatures plus impurity ion Doppler shifts for ion temperatures to measure the plasma energy content did this. Only the energy confinement of decaying spheromaks was measured. The new CORSICA capability will provide a framework in which to interpret data, including profiles when available, from SSPX.

HIT. On the HIT experiment at the University of Washington, 18 the code EFIT was modified to include open-field-line current with a separate $F F^{\prime}$ (measure of toroidal current effects and the prime indicates the derivative with respect to the flux) in the short-path open flux region. The long-path open flux and the closed flux region were modeled with the same polynomial for $F F^{\prime}$. The injector current, injector flux, excluded flux and surface poloidal magnetic probes were used as fitting data for EFIT. Only the closed flux has non-zero pressure derivitive, $P^{\prime}$. Stability analysis was done with the codes PEST and PEST3 developed at Princeton. With the plasma in contact with the conducting wall, PEST3 showed that the HIT equilibrium was stable when pressure was included. However, with less than a centimeter of vacuum space between the wall and the plasma, it was unstable to the $n=1$ mode that is observed. Thus, vacuum space between the plasma and wall is critical. Only extremely crude energy confinement time measurements were made on HIT using single-point Thomson scattering data for plasma energy and estimates of the Ohmic heating for the power input. The internal current density came from EFIT equilibrium code developed at General Atomics with a guess of 2 for the effective impurity atomic number. We know that the internal current density calculation is extremely inaccurate from EFIT since we had no internal magnetic diagnostics. 
As we approach the initial operation of SSPX and plan for analysis of new spheromak data, several uses of CORSICA will play important roles for understanding results and guiding experiments. In some cases, these will require extension of the computational capabilities:

- To interpret specific diagnostics. These include the USPR and the magnetic probes installed in the flux conserver.

These probes will be used to determine the experimental spheromak magnetic fields and current levels. In addition, they will be used to infer the profiles of the current in the plasma. Although considerable progress has been made, this inversion is still being worked on. Solution of this inversion using physically realizable spheromak parameters is the highest priority for CORSICA in the near term. It requires EFIT-like equilibrium fitting but with force-free currents on open field lines. CORSICA includes the required capabilities:

- $P^{\prime}=0$ and $\lambda=F^{\prime}$ are given as input boundary conditions on open lines.

- $P^{\prime}$ and $\lambda$ are fit to functions in closed-flux regions like EFIT.

- Short-path open flux is be treated differently from closed flux of same value.

- Ideal, resistive, ballooning, Mercier, and Hall linear 3D stability analyses of equilibrium with current on open field lines are needed. A vacuum edge regions option is needed where the equilibrium has $\Gamma^{\prime}=0$. Presently, only ballooning and Mercier are available; other stability analysis must be done using CORSICA equilibria as the starting point of a separate code. This stability analysis is presently in the code but does not work properly; making it useful is very important for comparing with experiment.

- To evaluate experimental processes in SSPX, especially those related to energy confinement and transport. An "analysis" package has been written for CORSICA, but as it has not been exercised extensively it will undoubtedly require further development before it can be reliably be used to interpret data in the context of spheromak physics. Some small code modifcations in the analysis mode are required to accommodate the new formulation of CORSICA based on poloidal flux (needed for both spheromak and RFP transport modeling).

A demonstration of the analysis mode in CORSICA using existing DIII-D data is planned. With the appropriate experimental data, onc also can deduce a current transport coefficient from a transport simulation run in the analysis 
mode. The nearest term application of this capability is likely to be to the RFP; see below.

- To provide equilibria for studies of resistive, time-dependent MHD, e.g. using the NIMROD code. (NIMROD is an obvious choice for this purpose because it has the appropriate physics and DOE is investing resources in its development and use.) Time-dependent MHD simulations should be 3D with a Ohm's law including Hall effects to capture relaxation physics (NIMROD has this.).

As NIMROD is very complex, full integration into CORSICA is unwarranted at this time. Calculations of the magnetic geometry can provide the basis for interpretation of the resistive MHD mode effects (magnetic "turbulence") which both drive the spheromak current by a magnetic dynamo and open magnetic-field surfaces thus allowing energy losses. This work will include the development of semi-emperical models of the dynamo and resistive MHD for inclusion in CORSICA. These models could initially be based on hyper-resistivity models, and extended as new results are developed.

- In a new computational effort, a LDRD project has started to apply the edge tokamak physics code, UEDGE, to SSPX. Although this modeling will be separate from CORSICA in the near term, UEDGE has been coupled to CORSICA for tokamak studies. It is anticipated that when progress warrants, edge-core coupling will be extended to spheromaks to provide greater computational capability.

\section{Proposed Applications of CORSICA to Reversed Field Pinches (RFPS)}

A natural extension of CORSICA is to the RFP. Much of the physics is similar to the spheromak, and computational support for the RFP needs extension as experiments address new physics such as profile measurements for transport studies. Most of these applications are similar to those needed for SSPX. Applications requiring little or no modification of the code include:

- The starting point for CORSICA applications of interest to the Wisconsin RFP group is to produce equilibrium calculations for the Madison Symmetric Torus, MST ${ }^{19}$ using MST specifications for their device (coil geometry, limiter dimensions, etc.). Initial CORSICA equilibrium calculations have already been done for MST, and these can easily be extended to include additional physics.

- Perhaps the most important and immediate modeling need of the Wisconsin group in understanding MST results, that is a good fit to CORSICA capability, is to back out energy and particle transport/diffusion coefficients from profile measurements to be made on the experiment (density, temperatures, current, etc.). This corresponds to the analysis mode of 
operating CORSICA. Not much effort will be needed to modify the CORSICA analysis mode for RFP applications, and this should be given a high priority.

If CORSICA cannot undertake this function then the MST group would have to use two transport codes if they wanted to use CORSICA for anything else.

- Time-dependent equilibrium and diffusion studies to evaluate the diffusion of magnetic flux through the poloidal gaps in the the MST limiter can be undertaken by CORSICA using TEQ with a free boundary. If the experimental loop voltage is used as input, the poloidal variation of the MST equilibrium can be straightforwardly modeled in CORSICA.

- MST is considering the addition of a vertical magnetic field and additional correction of field errors. In one scenario, MST would acquire internal coils, which would be easy to model in CORSICA. The effects of the MST iron core are of interest in the consideration of vertical field issues and field error corrections. CORSICA calculations can address the quantitative consideration of the iron core within an axisymmetric framework with some code modification. CORSICA will describe the effects on the equilibrium and can be used as a design tool for the iron core and coil currents. This should be another straightforward application of CORSICA requiring minor code modifications.

- Wisconsin would like to have a survey of the effects on ballooning stability (Mercier criterion) of shaped cross-sections in MST. This would allow an optimization study to be undertaken (optimizing profiles for density, temperature, safety factor, and cross section) and would contribute potentially to improved operating scenarios. This is another relatively easy application of CORSICA. It would be appropriate for LLNL to set up such a study and train a Wisconsin researcher to carry out the details. The Mercier limit for the first CORSICA MST equilibrium calculated has been evaluated, yielding a beta limit of .09 for an external magnetic field of $0.1 \mathrm{~T}$.

There are several applications which would require some additional extensions to the code, which would provide significant scientific payoff with relatively small effort:

- The Wisconsin group is interested in the calculation of current-drive scenarios and to assess issues related to current-profile control. Current profile control is very important in RFPs because it would be desirable to stabilize or at least moderate sawteeth, dynamos and magnetic turbulence, which presently limit confinement. Appropriate source(s) are needed to model the current drive from rf, e.g., ICRH or Alfven waves, or from oscillating field current drive (OFCD). OFCD employs oscillating fields that drive currents at the plasma edge, and the process by which the current is driven and penetrates the plasma would require some model of hyperresistivity or anomalous current transport, similar to those needed for the 
dynamo in the spheromak. As part of the optimization of the current drive, the degradation of current drive due to trapped particle effects needs to be included.

- Fully predictive, self-consistent modeling of RFP plasmas using CORSICA or any other code requires incorporation of some model or detailed simulation of the magnetic turbulence and dynamo physics in RFP plasmas. Modeling of this physics might be accomplished in CORSICA by solving for the equilibrium in conjunction with Ohm's Law using a hyper-resistivity (Strauss' or Boozer's model fitted to MST experimental data or a new model from Mattor for global modes) to model magnetic turbulence and dynamo action. Wisconsin presently relies on the Schnack's cylindrical timedependent non-ideal MHD code DEBS or the toroidal code TRIM. The new NIMROD code will augment or supercede TRIM. CORSICA would have to use a phenomenological model (e.g., modified Rechester-Rosenbluth or Terry model) or marry to TRIM or NIMROD as in CORSICA3.

Before proceeding, it is important to define a compelling and specific physics question that would be convincingly addressed by such simulations. Insofar as our spheromak modcling must confront the same questions of the effects of magnetic turbulence on confinement, and we would like to have CORSICA help us as much as possible in understanding and optimizing experimental plasma performance, LLNL needs to continue to investigate what can be done in this area. It was suggested that we contact Paul Terry at Wisconsin as well as continue dialogue with the NIMROD team. This research area is more open-ended than the ones mentioned in the foregoing and may require more modifications to CORSICA, but is clearly another significant area of opportunity.

- Connecting CORSICA with its BASIS interface directly to the MST database (as has been done already for the DIIID database) to facilitate modeling (including synthetic diagnostics) may be useful to the Wisconsin effort. Presently MST uses an older version of MDS for its data acquisition system that is based on VMS.

- UEDGE is an important LLNL modeling tool and is an integral part of CORSICA (CORSICA 2). Edge modeling is largely nonexistant for RFPs but will be important to RFP research sooner or later. RFPs operate without divertors today, but divertors will be required for a reactor. LLNL experience in edge plasmas and divertors could both be applied here, and is another potential area for collaboration with Madison.

The immediate strategy is for the LLNL and Wisconsin groups to come to an agreement on a list of possible modeling activities, establish priorities, and assess what can be done at LLNL and in collaboration with the Wisconsin group given available human resources. In many of the research activities outlined in the foregoing, LLNL can produce demonstrations of CORSICA 
capability or undertake minor code modifications and then demonstrate new capabilities and work with Wisconsin collaborators to teach them how to use CORSICA under current funding and programmatic obligations. Several of the applications requiring more significant extensions of CORSICA are similar to those for spheromaks, and can be undertaken as part of a collaborative effort.

\section{Program Directions}

The development of attractive magnetic confinement fusion alternate concepts is an important element in the U.S. magnetic fusion program and is receiving increased emphasis by DOE's Office of Fusion Energy Sciences (OFES). The LLNL MFE Program is undertaking the SSPX spheromak experiment currently jointly funded by LDRD and OFES. Our efforts in extending and applying CORSICA to spheromak calculations support our spheromak experiment and the OFES mission in alternate concepts. We will include a proposal to expand the development and application of CORSICA in our next Field Work Proposal to DOE with emphasis on spheromak, RFP, and spherical tokamak alternate configurations.

Our efforts in extending capabilities in the area of comprehensive and integrated modeling of magnetic fusion devices contribute to other new initiatives in the U.S. magnetic fusion program. As an outgrowth of a DOE 2000 proposal from the fusion community, OFES is sponsoring a new national collaboratory project entitled the National Transport Code Collaboration (NTCC). A principal goal of the project is to build a new, modern, general-purpose transport code with state-of-the-art physics content and a flexible, interactive user interface to support application steering. LLNL is a participant and leader in parts of the project, and is receiving new funding for this research activity. There is considerable common ground between our CORSICA projects and the NTCC activity.

There is a major new DOE Energy Research initiative to increase support for computational physics, the Scientific Simulation Program (SSP). Fusion is competing with other ER research programs for a significant level of new funding. OFES continues to support a strong theory program nationally (total budget $\sim \$ 18 \mathrm{M})$. The extension of CORSICA to support alternates logically fits into both the SSP and OFES theory program portfolios.

To acquire the appropriate physics content for a range of alternate fusion concepts, to enlist good help from other research centers, and to establish a signficant user community for our new software beyond the confines of LLNL, we have established several new collaborations in the last year with researchers who are expert in alternate fusion concepts. We are heavily involved with the rest of the fusion community in the NTCC and in drafting a magnetic fusion SSP proposal. In the alternates area, we are collaborating with University of Washington and University of Wisconsin (Madison) 
researchers in extending CORSICA to spheromaks and RFPs. We have also teamed with General Atomics and Wisconsin researchers in a new proposal to model the linear and nonlinear resistive MHD stability of spheromaks. This work will involve codes that will be interfaced with CORSICA.

In summary, there are a number of avenues to follow in extending the capabilities of CORSICA to model alternate fusion concepts; and there are real opportunities for new funding to support this activity.

\section{Acknowledgments}

The authors thank J.A. Crotinger (now at Los Alamos), T.A. Casper, and other members of the Magnetic Fusion Program at the Lawrence Livermore National Laboratory for their assistance in this research.

\section{References}

1. G.B. Zimmerman and W.L. Kruer, Comments Plasma Phys. Control. Fusion 2, 51 (1975).

2. P.F. Dubois, "Making Applications Programmable", Comp. Phys. 8, No. 1 (1994); P.F. Dubois and Z.C. Motteler, Basis Language Reference Manual, Technical Report UCRL-MA-118543, Lawrence Livermore National Laboratory, Livermore, CA, 1994.

\section{L.L. LoDestro and L.D. Pearlstein, Phys. Plasmas 1, 90 (1994).}

4. J.A. Crotinger, L.L. LoDestro, L.D. Pearlstein, A. Tarditi, T.A. Casper, and E.B. Hooper, "CORSICA: A Comprehensive Simulation of Toroidal MagneticFusion Devices," Technical Report UCRL-ID-126284, Lawrence Livermore National Laboratory, Livermore, CA, 1997.

5. D.A. Humphreys, J.A. Leuer, A.G. Kellman, S.W. Haney, R.I. Bulmer, and L.D. Pearlstein, Fus. Technol. 26, 331 (1994).

6. T.A. Casper, J. Crotinger, S. Haney, J.M. Moller, L.D. Pearlstein, B.W. Rice, and B.W. Stallard, "Status report on time-dependent modeling for currentprofile feedback control, Technical Report UCRL-ID-122226, Lawrence Livermore National Laboratory, Livermore, CA, 1995; T.A. Casper, J. Crotinger, J. Moller, L.D. Pearlstein, B.Rice, B. Stallard, L. Lao, and T. Taylor. "Modeling of current-profile evolution and equilibria in negative-centralshear discharges in the DIII-D experiment, in 23rd European Conference on Controlled Fusion and Plasma Physics: Kiev, Ukraine, June 24-28, 1996, vol. 
20C, part I of Europhysics Conference Abstracts, p. 295, European Physical Society, 1996.

7. T.D. Rognlien, B.J. Braams, and D.A. Knoll, Contrib. Plasma Phys. 36, 105, (1996).

8. A. Tarditi, R.H. Cohen, G.G. Craddock, J.A. Crotinger, G.D. Porter, T.D. Rognlien, A.I. Shestakov, and G.R. Smith, Contrib. Plasma Phys. 36, 132, (1996).

9. T.A. Casper, B.I. Cohen, J.A. Hawreliak, L.L. LoDestro, and W.H. Meyer, "Synthetic Diagnostics for Modeling Magnetic Fusion Experiments," Technical Report UCRL-ID-13000, Lawrence Livermore National Laboratory, Livermore, CA, 1998.

10. B.I. Cohen, E.B. Hooper, M. C. Spang, and C.W. Domier, "Theoretical Aspects of the Use of Pulsed Reflectometry in a Spheromak Plasma," Rev. Sci. Instrum., to be published, Technical Report UCRL-JC-129499, Lawrence Livermore National Laboratory, Livermore, CA, 1998.

11. R.H. Cohen, E.B. Hooper, L.L. LoDestro, N. Mattor, L.D. Pearlstein, D.D. Ryutov, "Theoretical Issues in Spheromak Research" Technical Report UCRL-ID-127002, Lawrence Livermore National Laboratory, Livermore, CA, April 1, 1997.

12. E.B. Hooper, "Coupled Spheromak-Helicity Injector in the Sustained Spheromak Physics Experiment, SSPX," Proceedings of the US-Japan Workshop: Physics of High-Beta Fusion Plasmas, University of Washington, Seattle, Washington, March 18-20, 1998, pp. 39-42.

13. E.B. Hooper, L.D. Pearlstein, and R.H. Bulmer, "MHD Equilibria in a Spheromak Sustained by Coaxial Helicity Injection," to be submitted to Nucl. Fusion.

14. R. Gatto, A.D. Turnbull, and E.B. Hooper, "Equilibrium and Stability Studies for the Sustained Spheromak Physics Experiment, SSPX," Bull. Am. Phys. Soc. 42, 1915 (1997).

15. D.D. Hua, E.B. Hooper, and T.K. Fowler, "Modeling Electron Heat Transport During Magnetic Field Buildup in SSPX," Technical Report UCRLID-128656, Lawrence Livermore National Laboratory, Livermore, CA, October 1997.

16. T.K Fowler, D.D. Hua, E.B. Hooper, R.W. Moir, and L.D. Pearlstein, "Pulsed Spheromak Plasma," Rev. Sci. Instrum., to be published.

17. S.O. Knox, et al., Phys. Rev. Lett. 56, 842(1986). 
18. T. R. Jarboe, M. A. Bohnet, A. T. Mattick, B. A. Nelson, and D. J. Orvis, Phys. Plasmas 5, 1807 (1998).

19. B. E. Chapman, et al., Phys. Plasmas 5, 1848 (1998). 


\section{Alternate approaches to magnetic fusion require advanced physics}

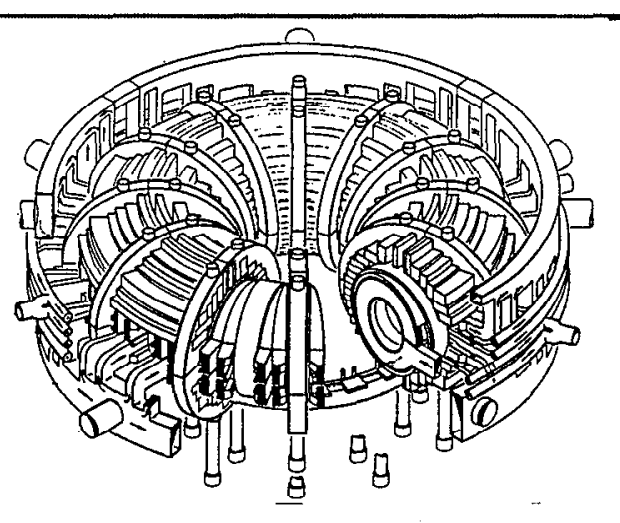

- Spheromak

- Current driven by magnetic dynamo with no external toroidal field

- Reversed-field pinch (RFP)

- Current driven by magnetic dynamo in external toroidal field
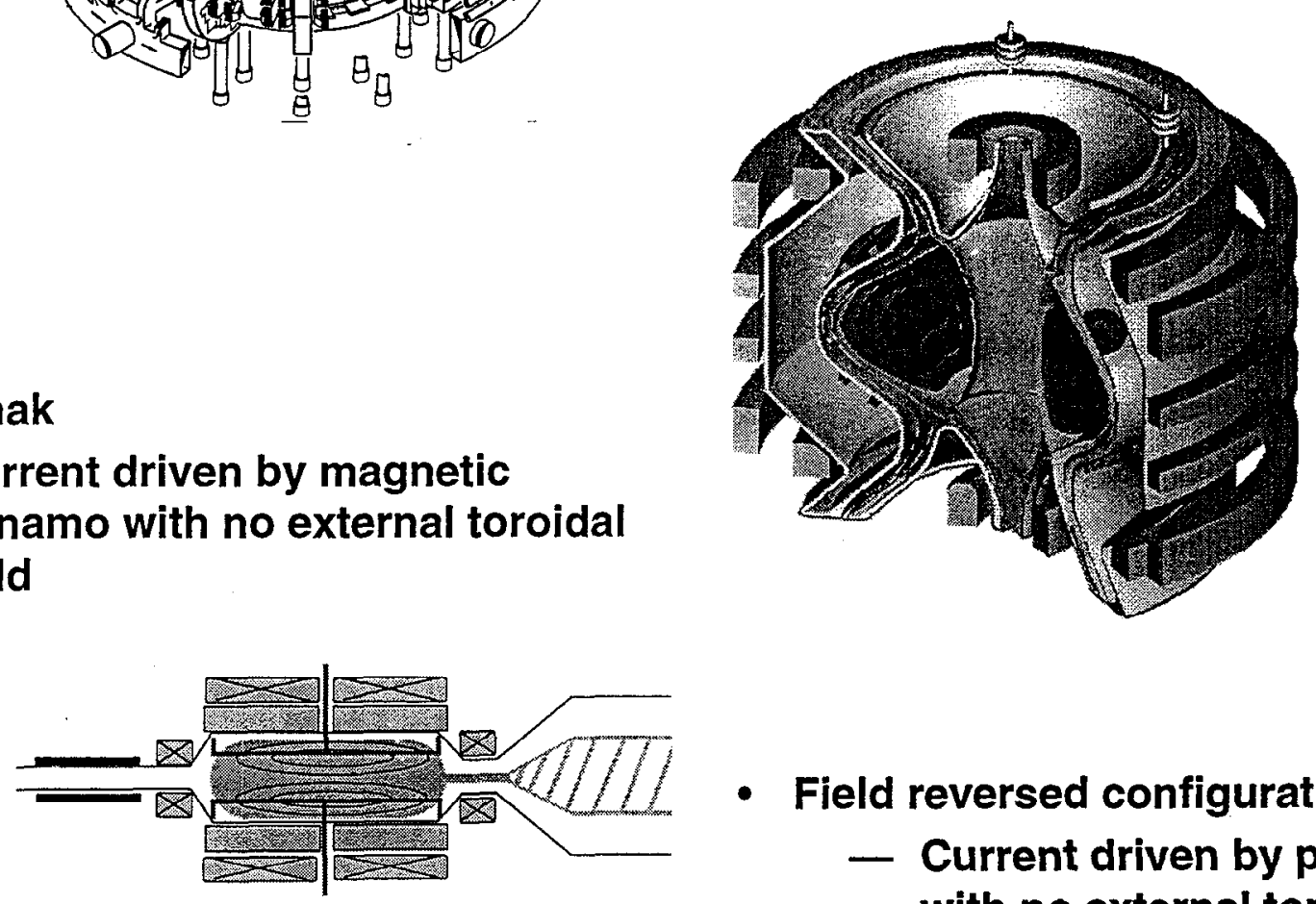

- Field reversed configuration (FRC)

- Current driven by pressure gradients with no external toroidal field 


\section{CORSICA's blueprint for a numerical tokamak.}

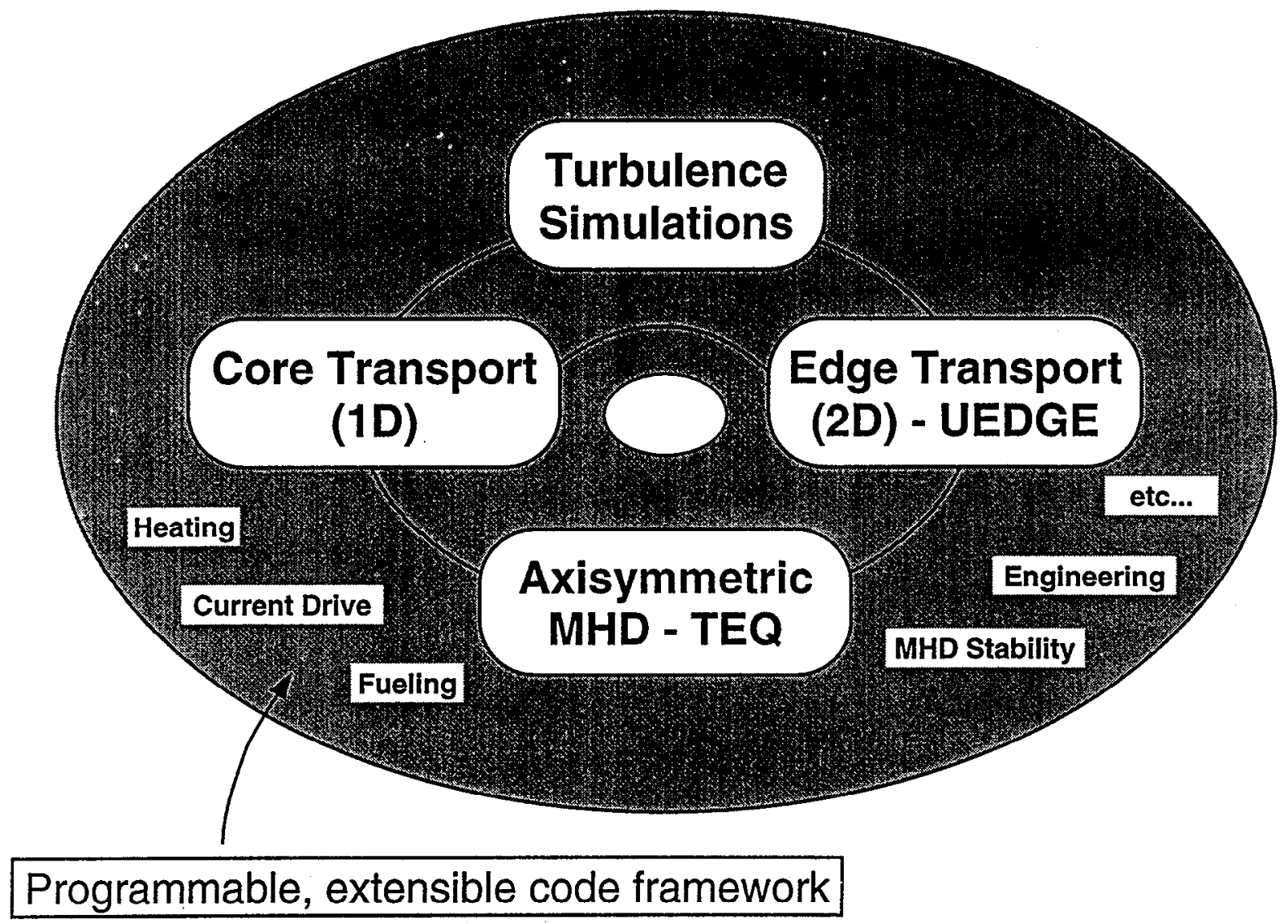

Figure 2. Schematic of the CORSICA integrated magnetic fusion modeling code. 
CORSICA models core and edge transport, and the interaction of the plasma with external fields.

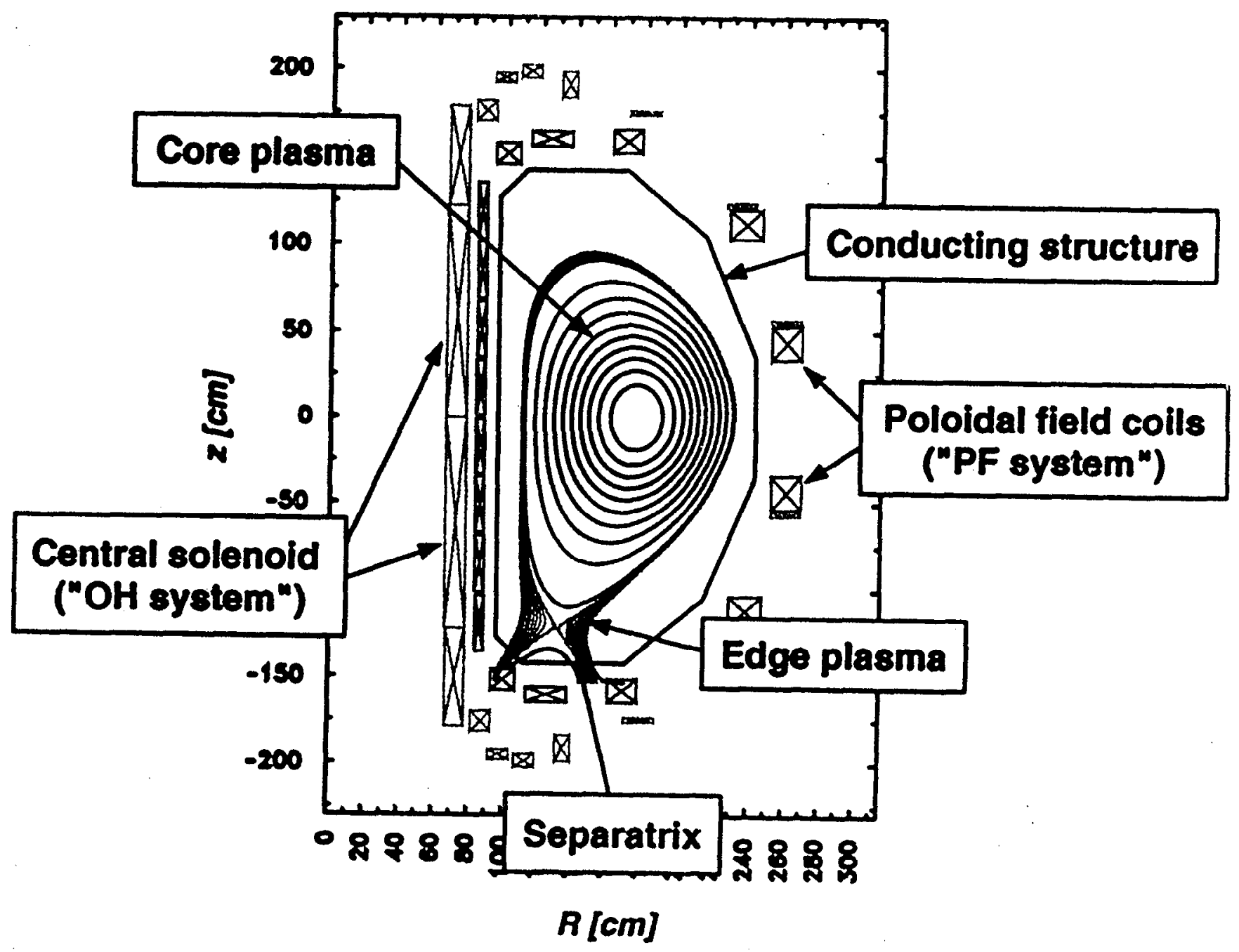


CORSICA 1 Calculated MHD Equilibrium for ITER Showing Magnetic Flux Contours and Coils.

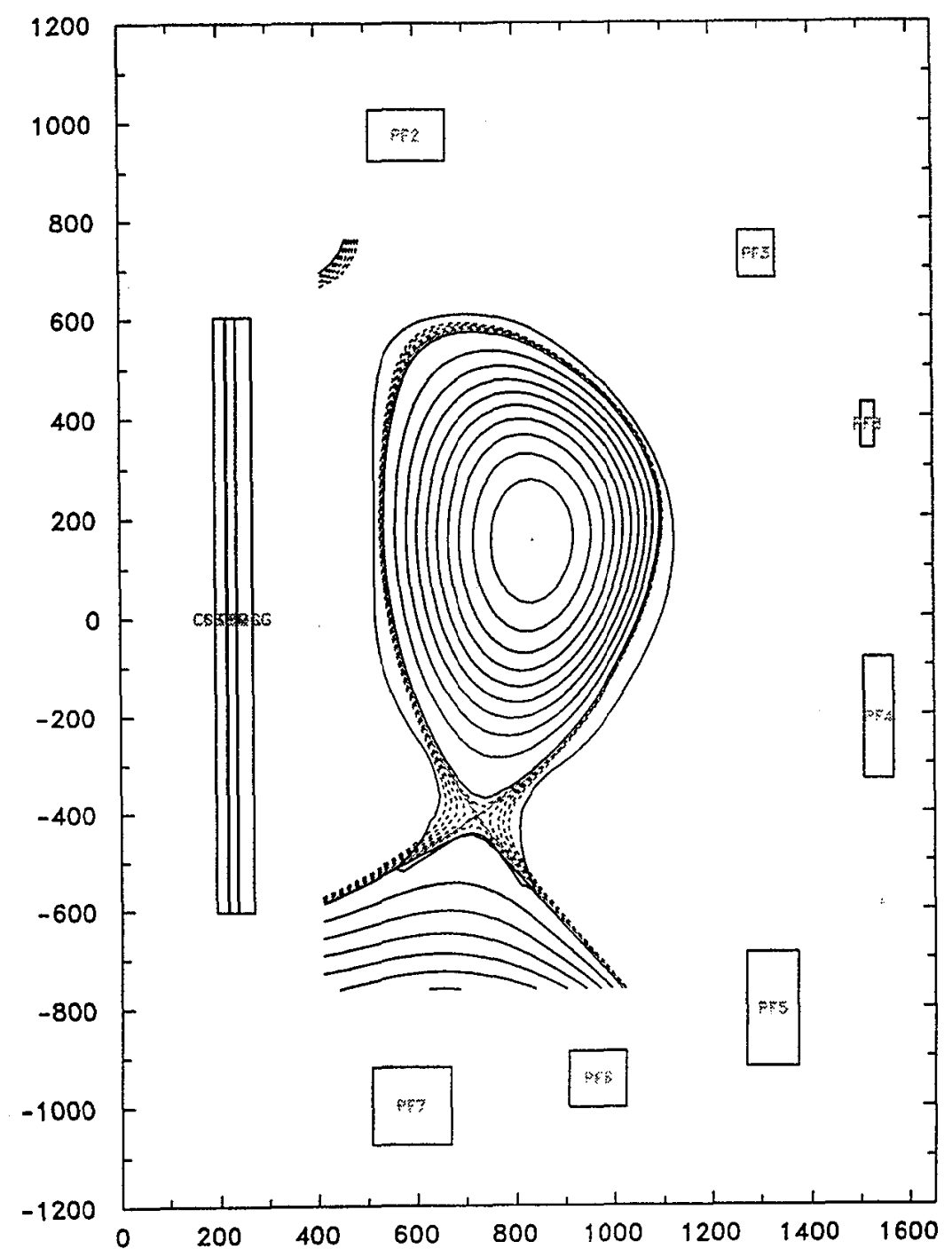

Figure 4. ITER flux surfaces and poloidal flux coil system calculated by CORSICA 1. 


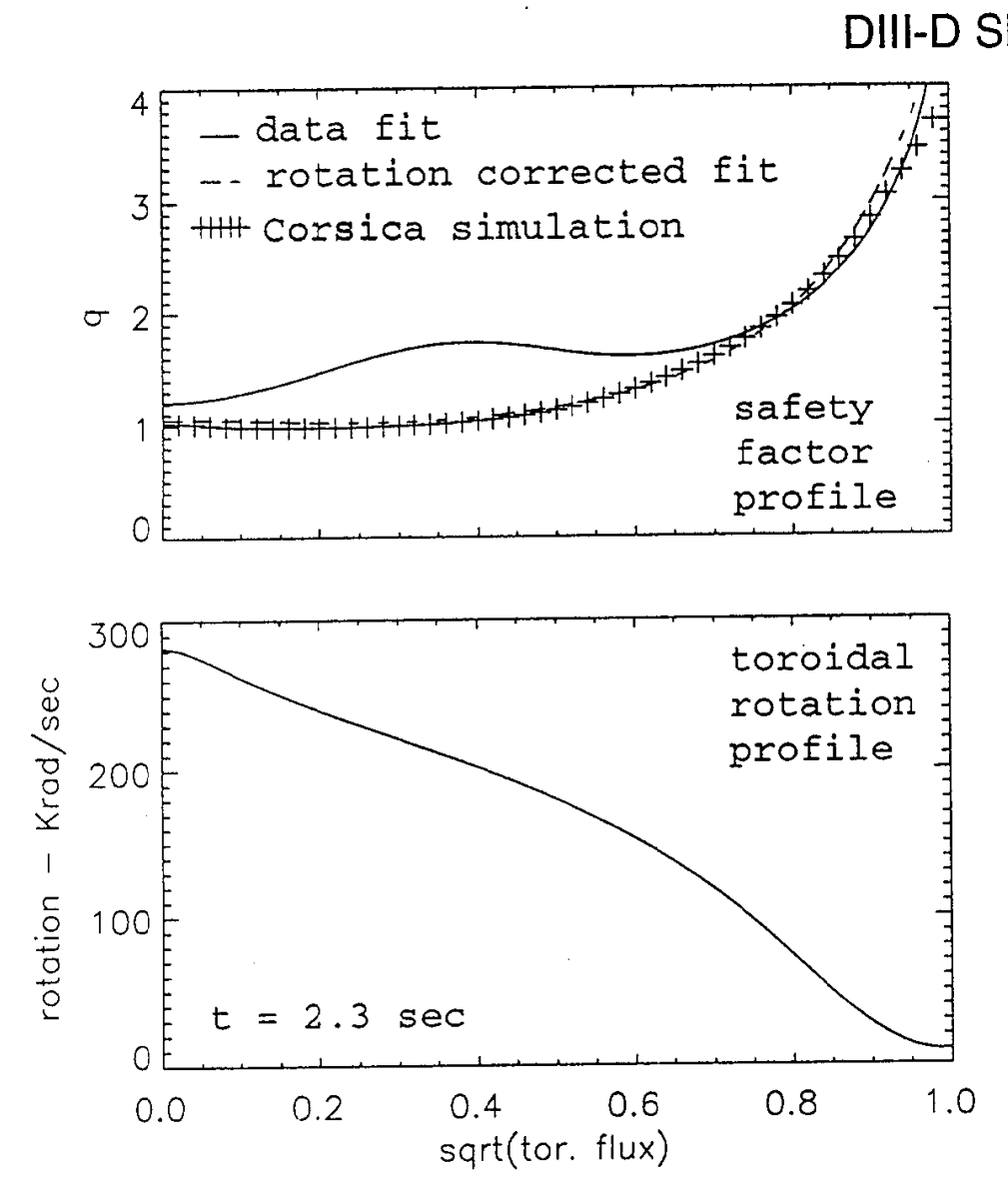

Shot 88964

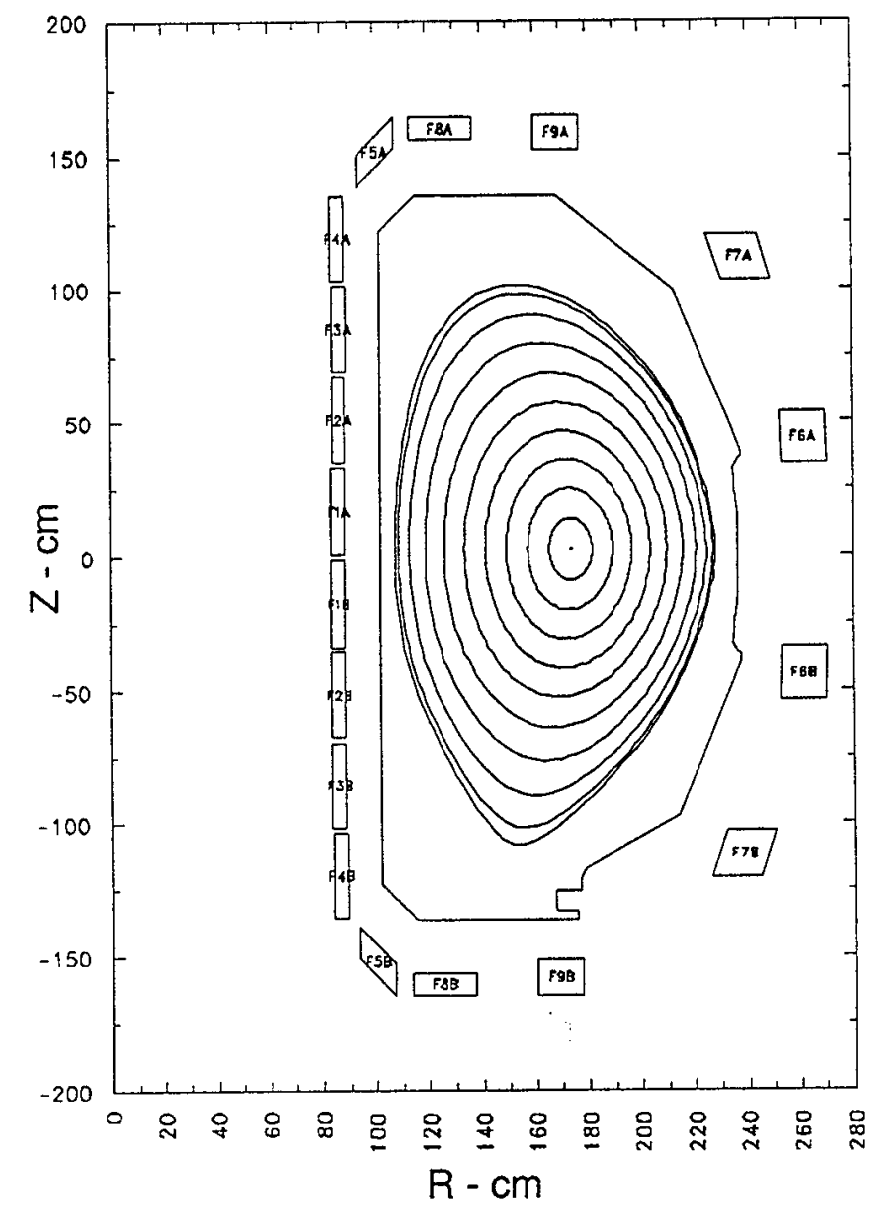

Figure 5. CORSICA 1 application to DIII-D shot \#88964 and comparison to experiment: magnetic equilibrium. 

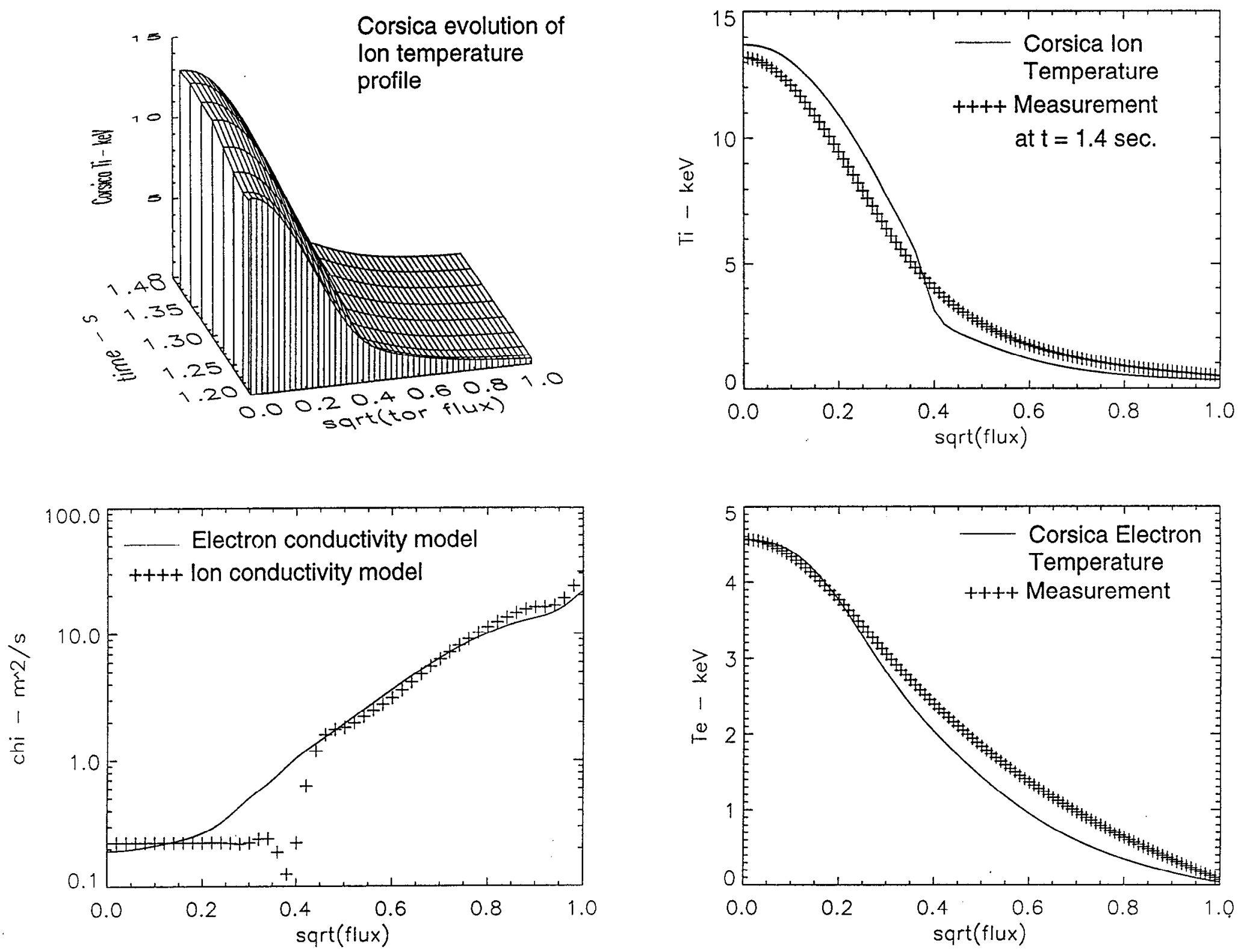

Figure 6. CORSICA 1 application to DIII-D and comparison to experiment: electron and ion temperature profiles. 
CORSICA 2: Modeling the core and the edge.

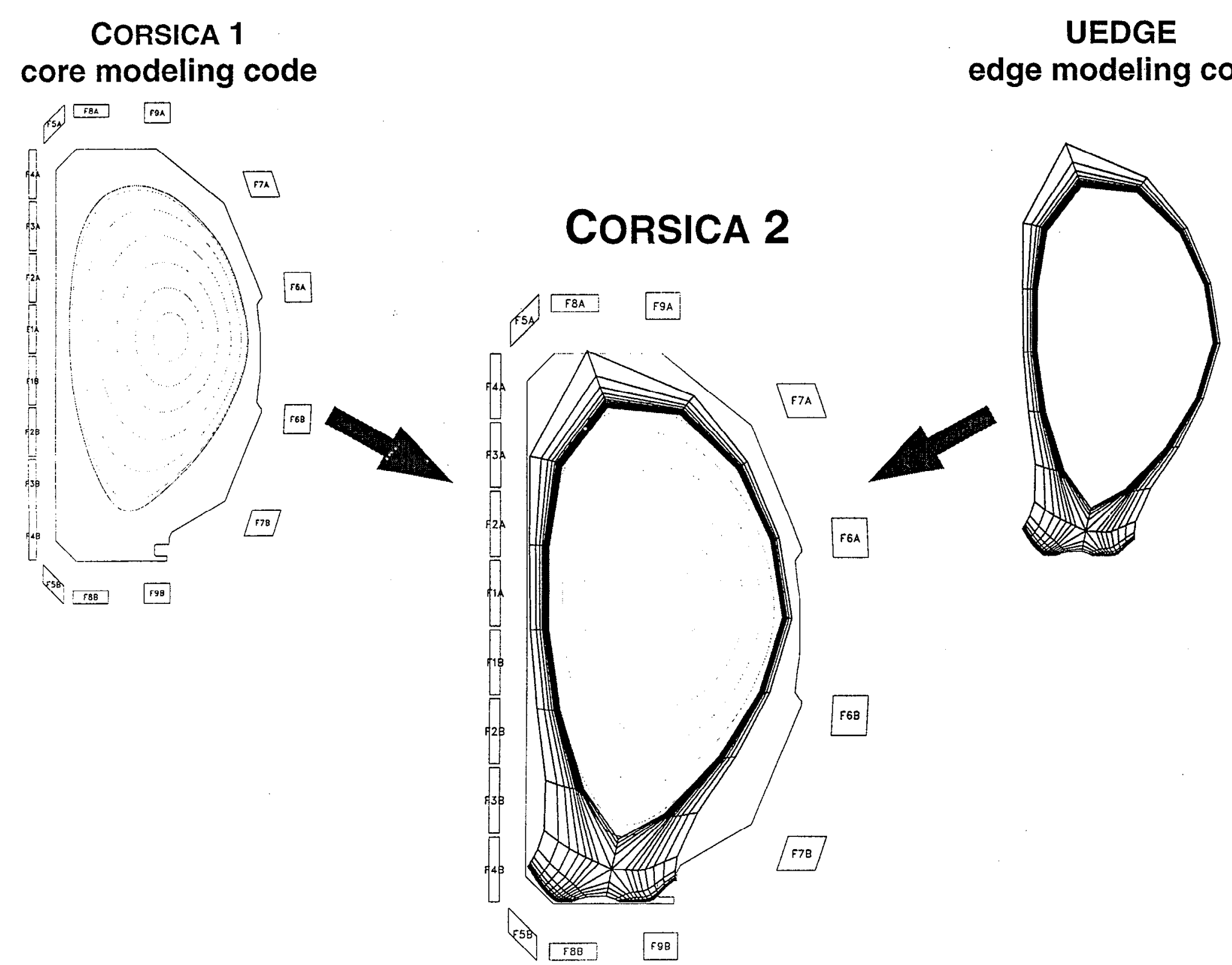

Figure 7. CORSICA 2 schematic. 
CORSICA 2 Calculated Electron Temperature Profiles Before and After Transition from Low to High Confinement Tokamak Mode.

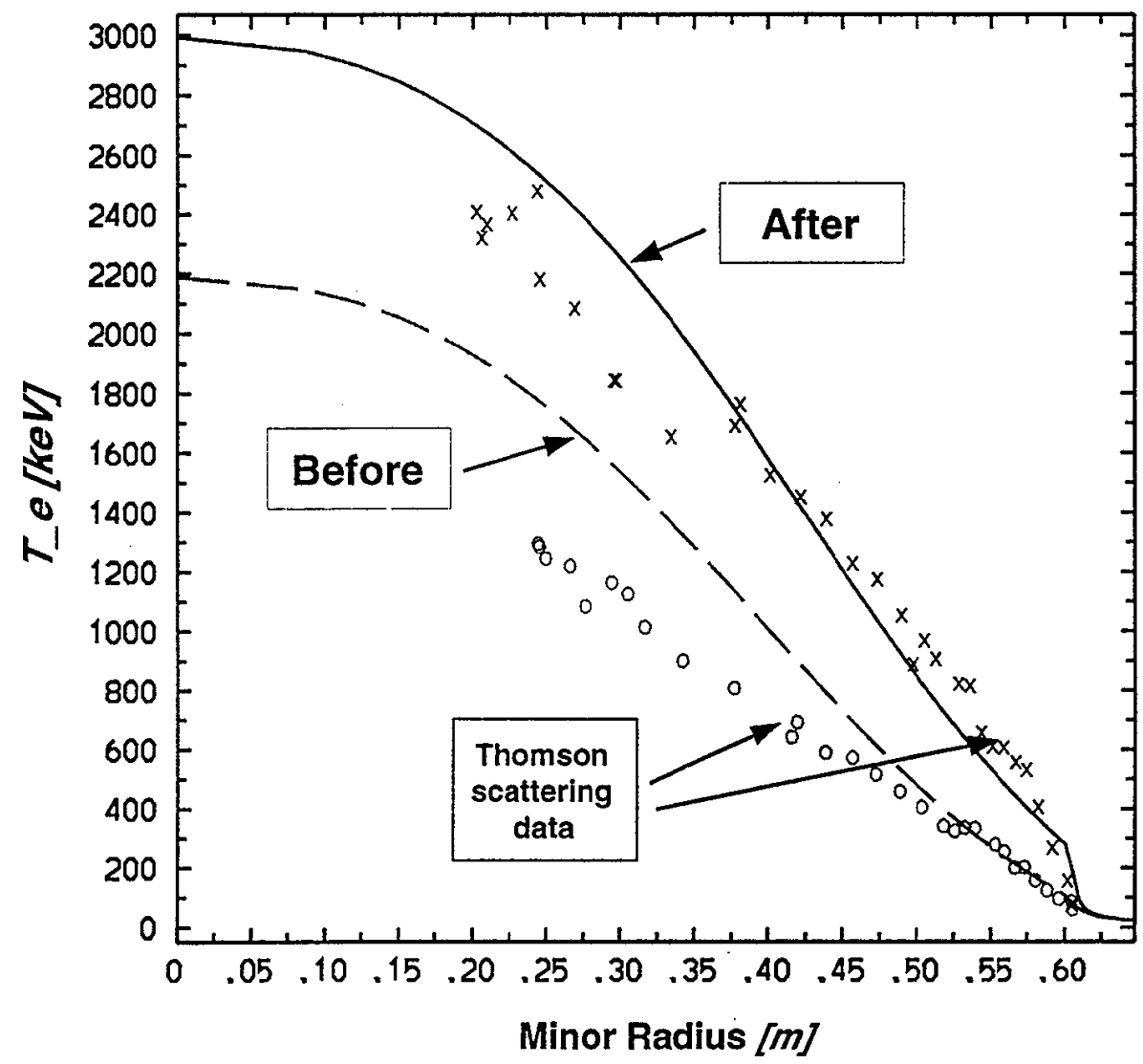

Figure 8. Electron temperature profiles before and after a transition from Low to High confinement mode modeled in CORSICA 2. 


\section{D O-Mode Reconstruction of $n_{e}(x)$ for SSPX Using CORSICA}

- CORSICA is used to calculate the equilibrium for the SSPX spheromak experiment being built at LLNL. The midplane electron density $n_{\theta}(x)$ and magnetic profile $\mathrm{B}(\mathrm{x})$ are then used in the reflectometry simulation from which $n_{e}(x)$ is reconstructed by Abel inversion:

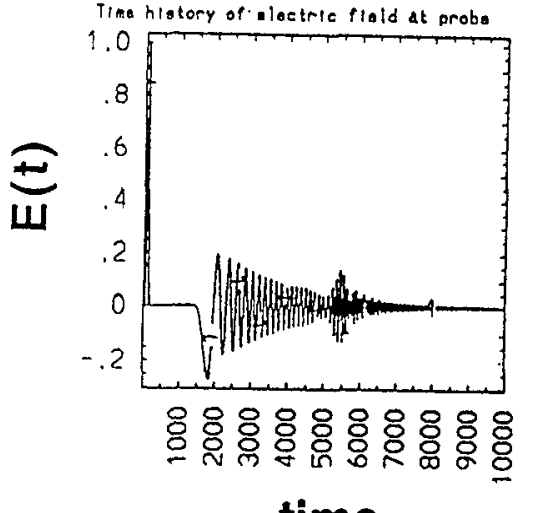

time

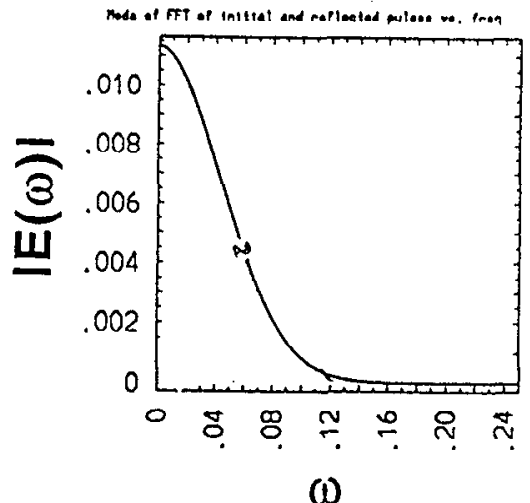

$\omega$

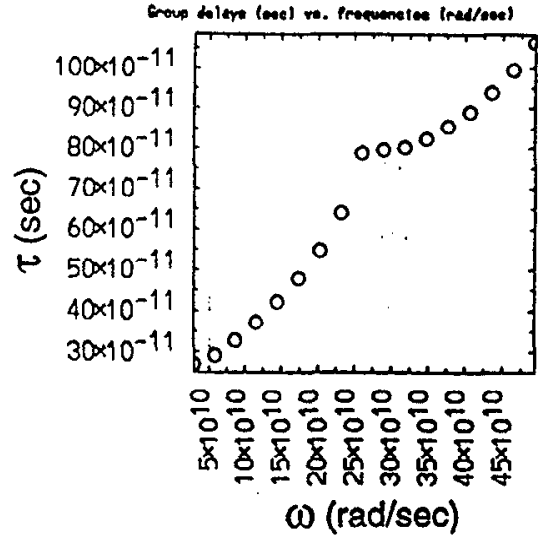

$\omega(\mathrm{rad} / \mathrm{sec})$

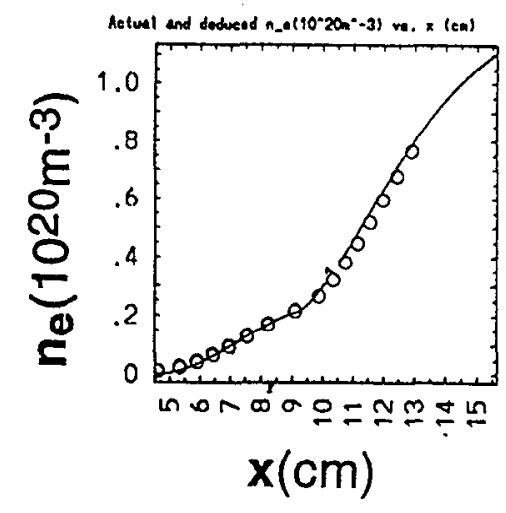

Figure 9. Simulation of ultra-short-pulse reflectometry applied to an SSPX model equilibrium. An ordinary-mode simulation is shown: CORSICA equilibrium, electric field at the antenna vs. time, reconstruction of the electron density profile. 
- CORSICA is used to calculate the equilibrium for the SSPX spheromak experiment being built at LLNL. The midplane electron density $n_{e}(x)$ and magnetic profile $B(x)$ are then used in the reflectometry simulation:
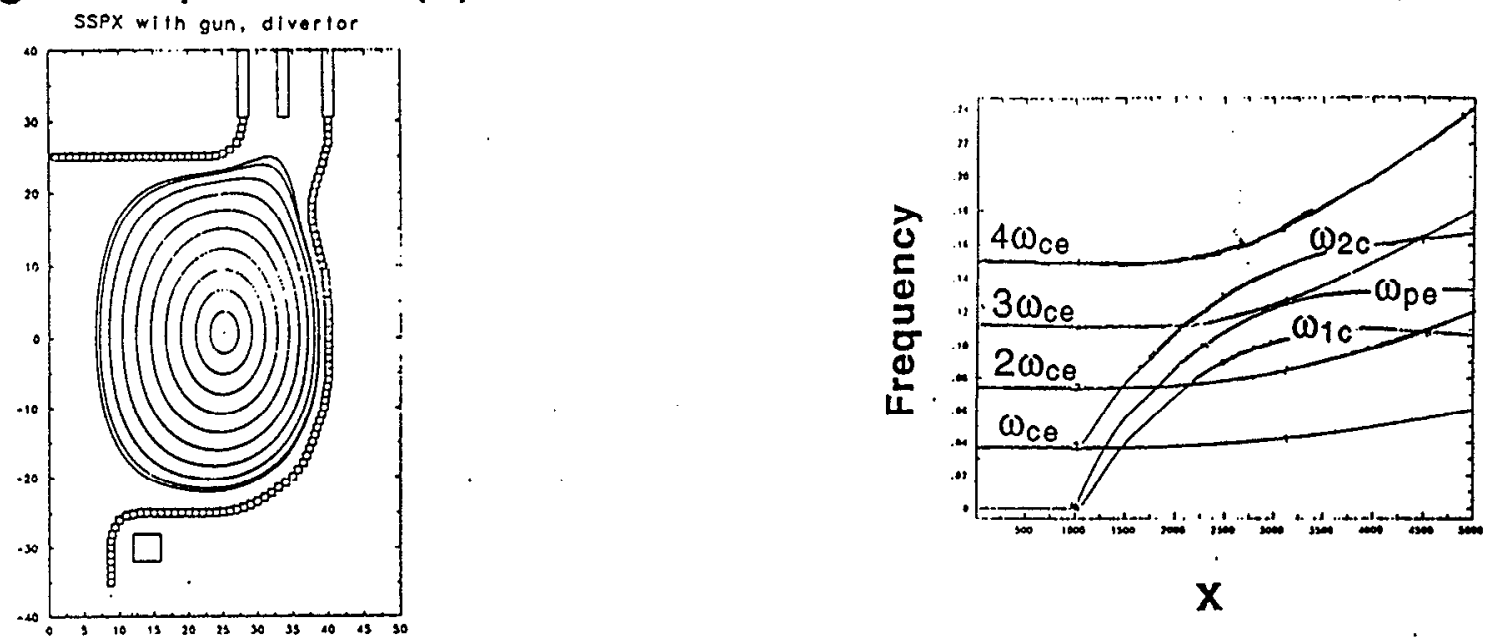

- X-mode reflectometry simulation:

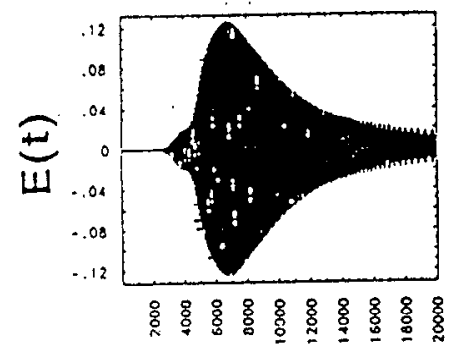

time

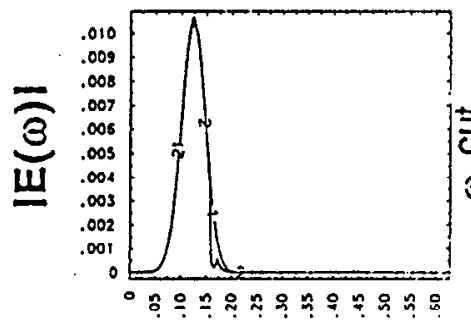

$\omega$

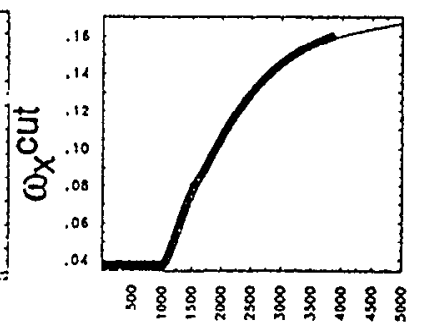

$\mathrm{X}$

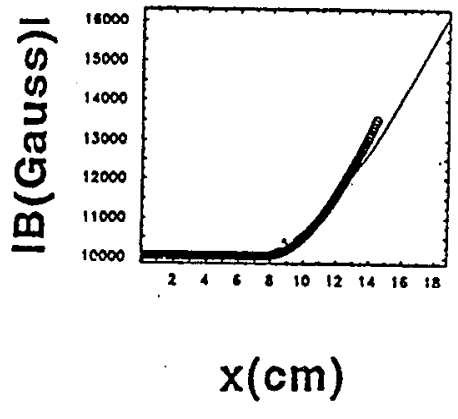

$x(\mathrm{~cm})$

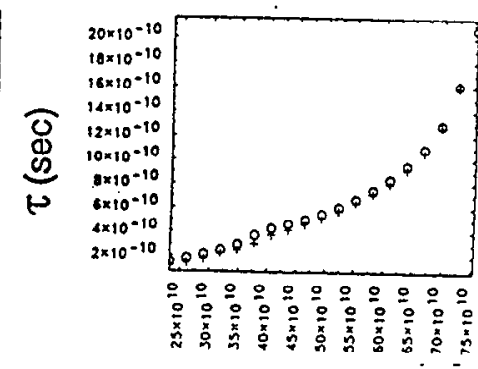

$\omega(\mathrm{rad} / \mathrm{sec})$

Figure 10. Simulation of ultra-short-pulse reflectometry applied to an SSPX model equilibrium. An extraordinary-mode simulation is shown: CORSICA equilibrium, cyclotron frequency and cutoff frequency profiles, reconstructions of the higher frequency $X$-mode cutoff profile, the measure and reconstructed group delays vs. frequency, and the reconstructed electron cuclotron frequency profile. 
CORSICA Synthetic Diagnostics: Emmissivity contours (left) for DIII-D shot \#84682 with soft X-ray chords overlaid for core chord(\#45) and edge chord(\#35). Comparisons (center) of simulated and measured data. Time-averaged rms error (right) between simulated and measured data for each detector.
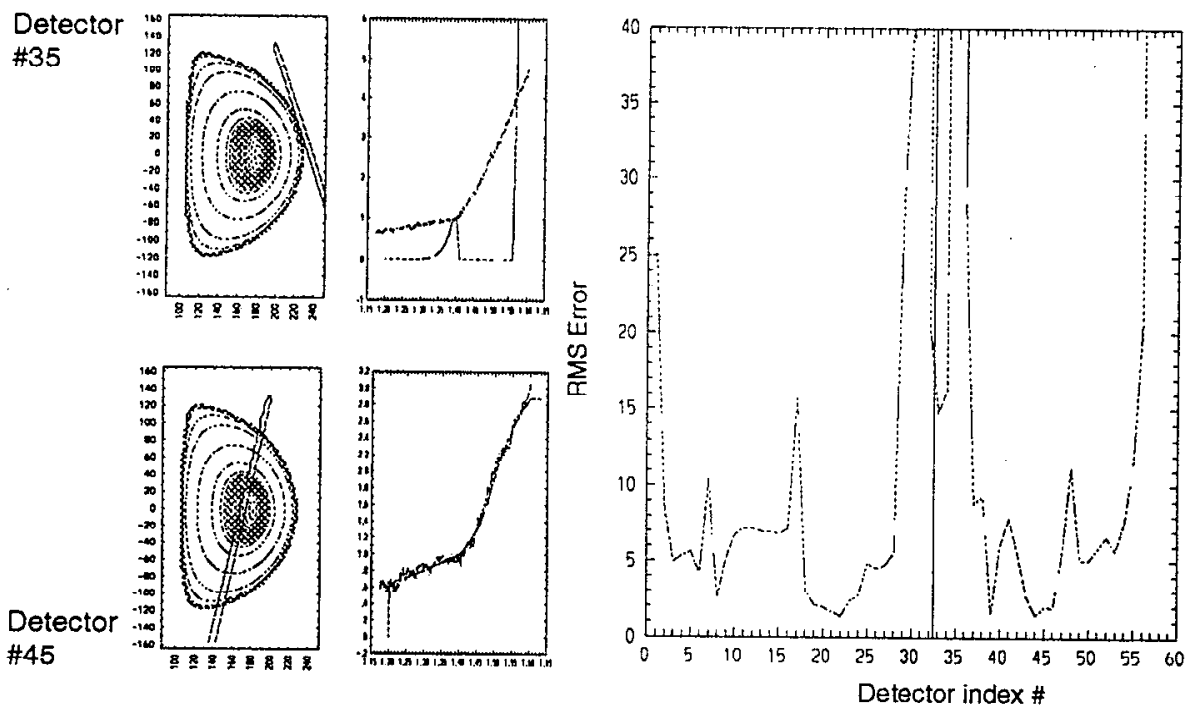

Figure 11. Simulation of the soft-Xray camera diagnostic on DIII-D shot \#84682. Emissivity contours with soft-Xray chords overlaid (left). Comparison of simulated and measured data (center), and rms errors for each detector (right). 
- Matching the WKB calculation of the linear mode conversion of $X \rightarrow O$ modes to reflectometry data provides a basis for a least-squares determination of the magnetic shear rate and pitch angle that is time and space-resolved.

$$
\left|\frac{E_{O}(\omega)}{E_{X}(\omega)}\right|=\left.\left|\int_{0}^{x} d x^{\prime} \frac{d \theta}{d x^{\prime}}\right| \frac{k_{X}}{k_{O}}\right|^{1 / 2} \exp \left[i \int_{0}^{x^{\prime}} d x^{\prime \prime}\left(k_{X}-k_{O}\right)\right] \mid \rightarrow \mathrm{d} \theta / \mathrm{d} x \text { and } \theta=\tan ^{-1}\left(\mathrm{~B}_{\text {tor }} / \mathrm{B}_{\text {pol }}\right)
$$

- From $\theta$ and $|B|$ profiles deduce $B_{\text {tor }}$ and $B_{p o l}$, and $\nabla \times B \rightarrow J$ vs. $x$

- CORSICA simulation of single and double-pass $X \rightarrow 0$ mode conversion in SSPX and reconstruction of $\mathrm{d} \theta / \mathrm{dx}$ and $\theta$ :
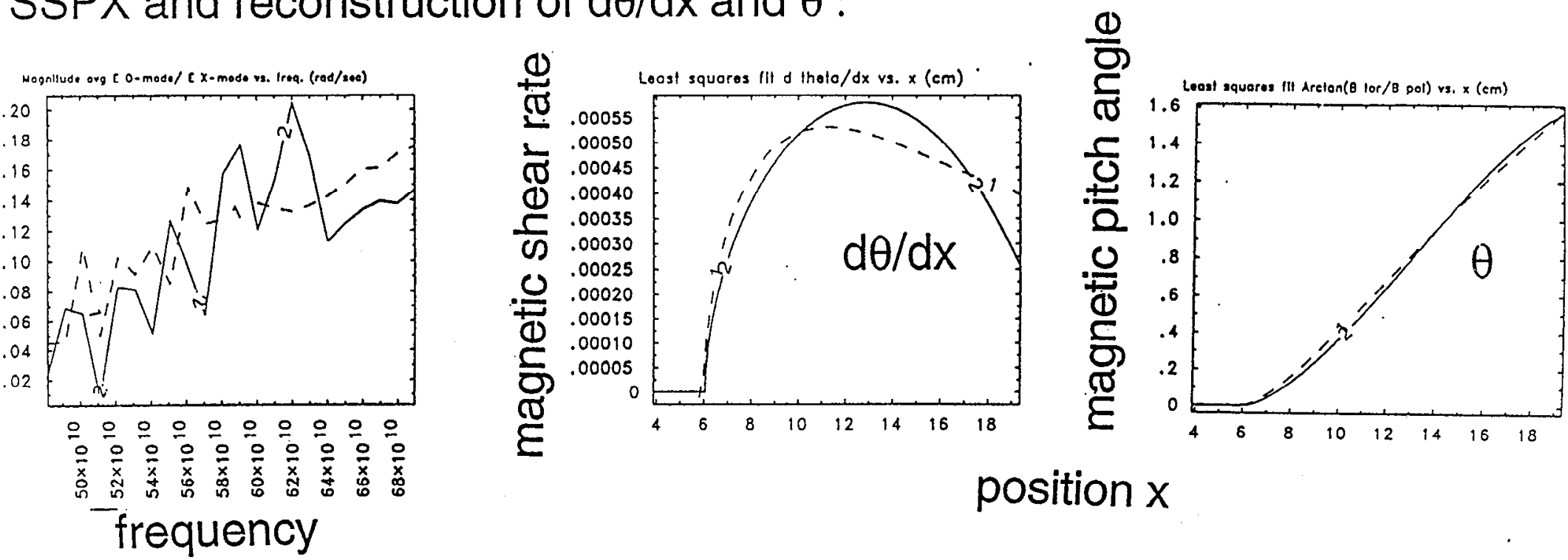

Figure 12. Comparison of simulation observations and the WKBJ-based leastsquares reconstructions of (a) the ratio of the mode-converted ordinary to extraordinary-mode amplitude as a function of frequency, (b) the magnetic pitch angle $\theta$ vs. $x$ and (c) $d \theta / d x$ vs. $x$. 


\section{Experimental design}

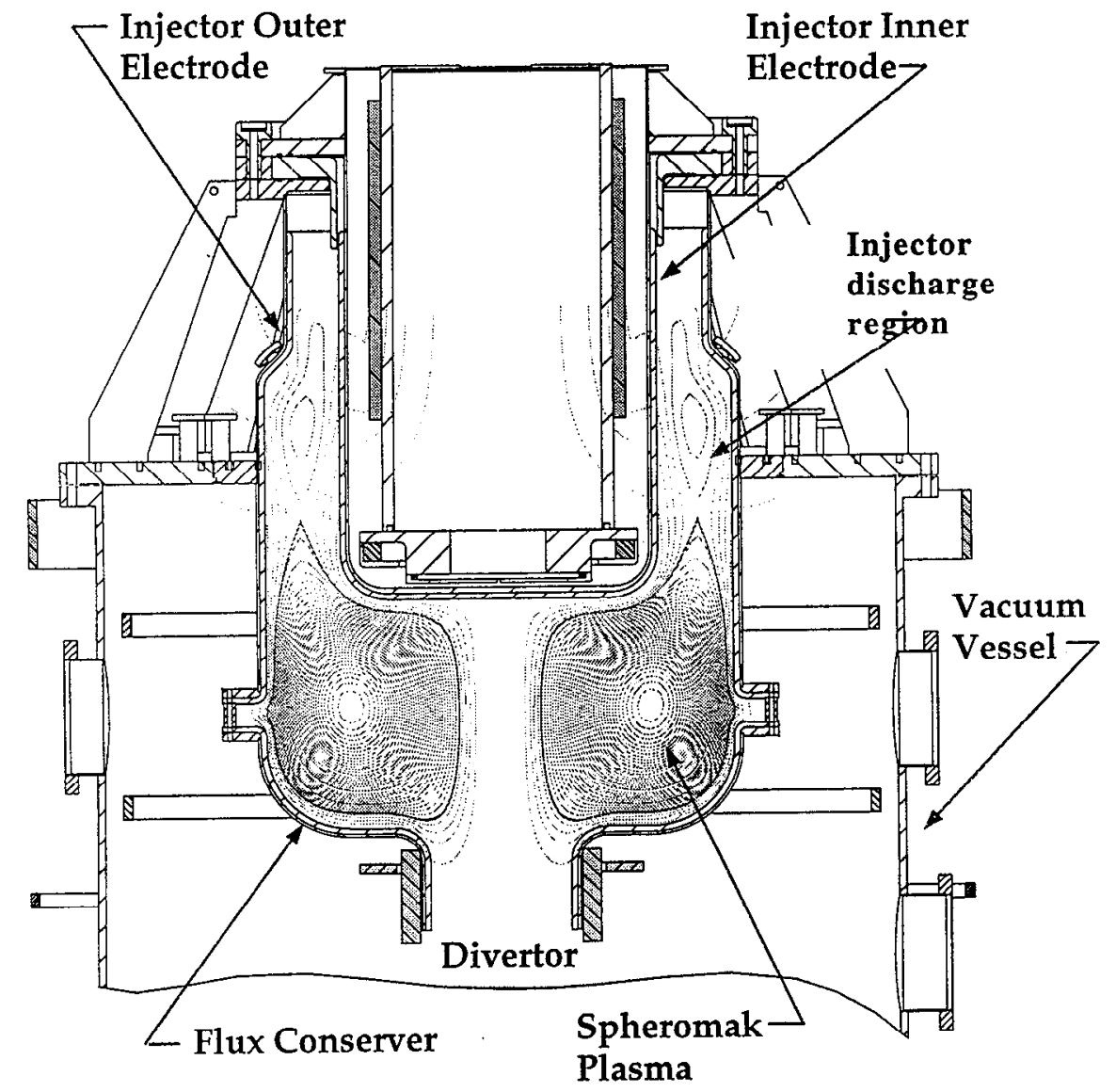

- Conformal solid flux conserver minimizes open field lines and associated dissipation

- Tungsten coating and boronization minimizes sputtering and impurities in the plasma

- Plasma minor radius $=23 \mathrm{~cm}$

- Expected peak $I_{\text {tor }} \approx 1.2 \mathrm{MA}$ or more, with $B \approx 1$ Tesla

Figure 14. CORSICA calculated magnetic equilibrium in the Sustained Spheromak Physics Experiment. 


\section{MHD equilibrium with force-free injector}

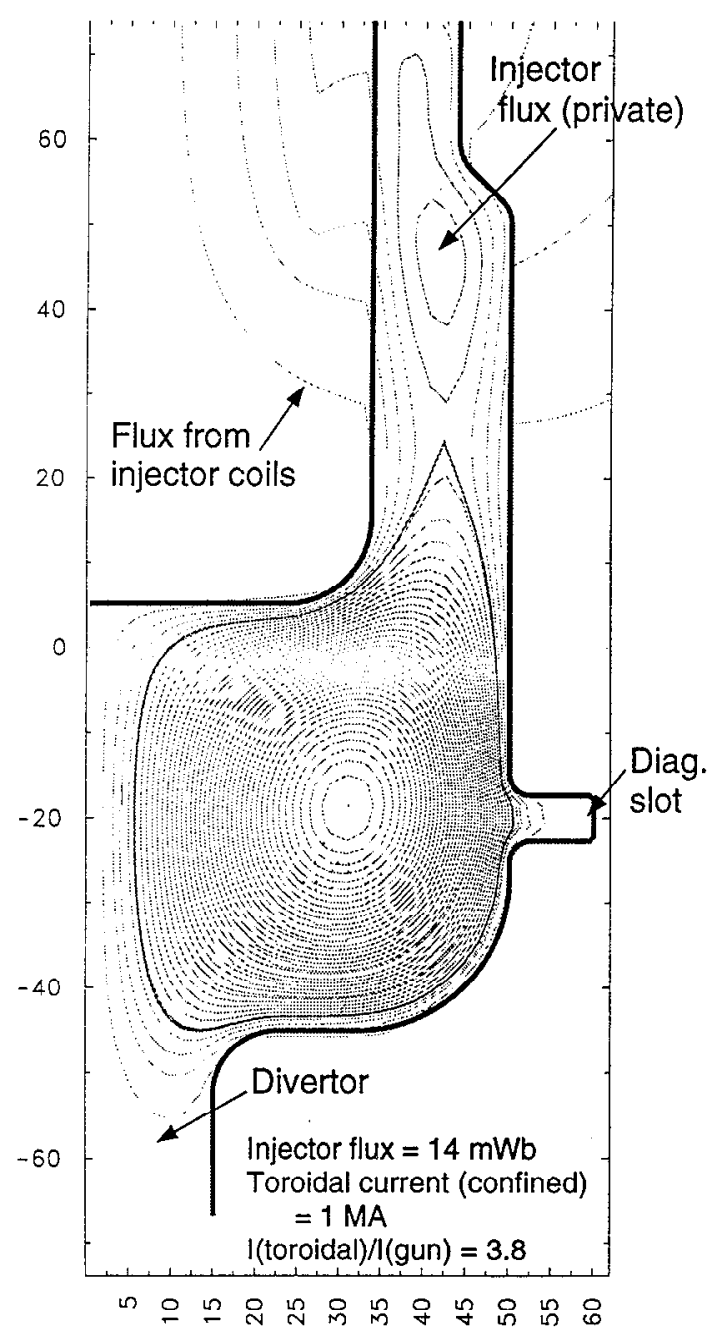

- The balance between edge and confined plasmas in the sustained spheromak depends on:

- Bias flux in the injector

- Injector current

- Toroidal (confined) current

- The ratio $\lambda_{\text {edge }} / \lambda_{\text {core }}$

- The interior profile of $\lambda$.

- The coupling between the edge current and the confined spheromak is determined by resistive MHD and is thus not well understood

-We can vary the parameters to determine goals to optimize the experiment 


\section{Magnetic field coil design for discharge control}

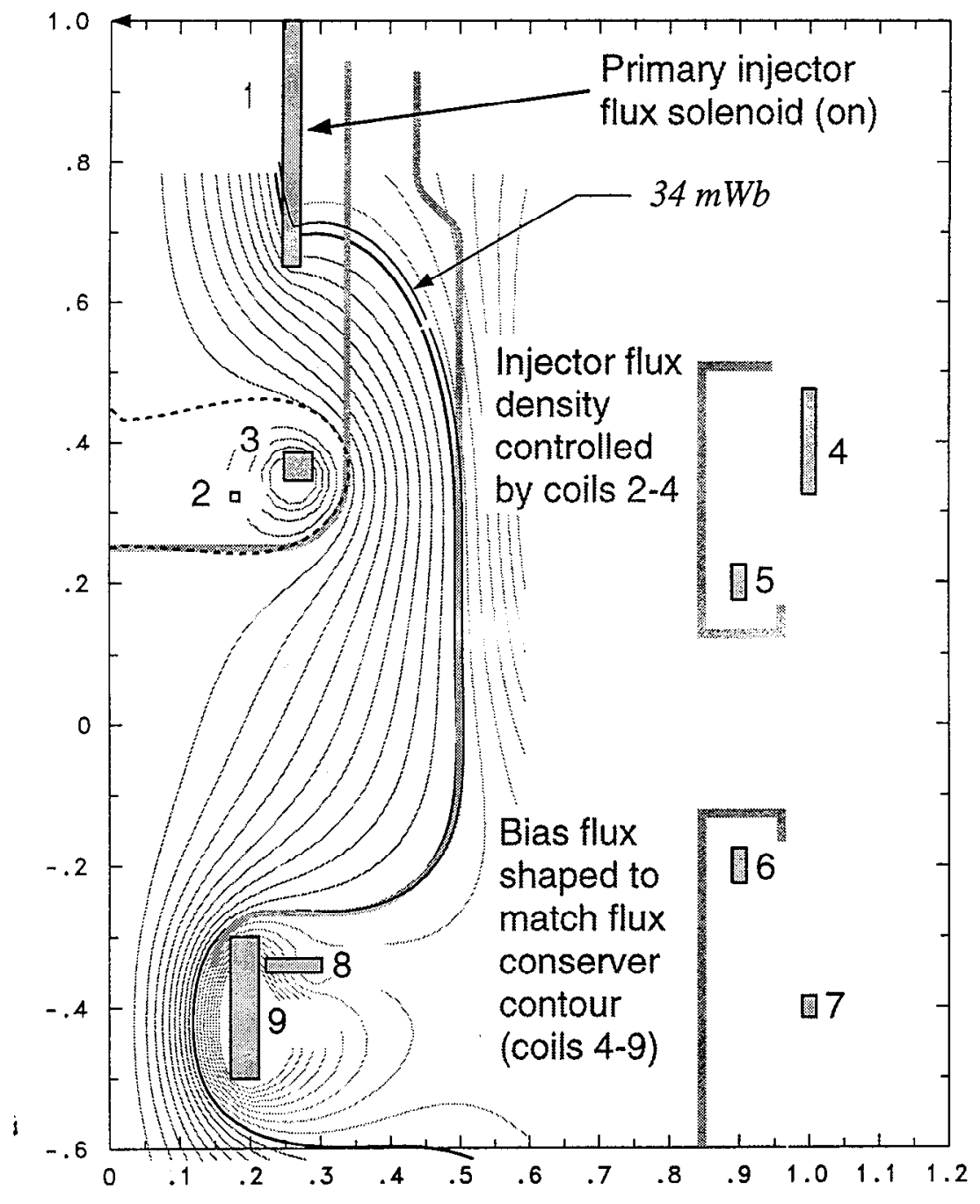

Coils are used to:

- Generate poloidal flux in the helicity injector ("gun")

- Generate a vacuum bias field to control the discharge configuration

Injector coils

- Designed to control the distribution of flux on the injector electrodes

Bias coils and flux conserver

- Vacuum magnetic field flux contour is tangent to the flux conserver to minimize field errors

Analysis by Dick Bulmer

Figure 16. CORSICA calculated magnetic field coil design and magnetic flux surfaces for discharge control. 


\section{Divertor configuration is controlled by bias coils}

- SSPX geometry and coils designed for flexible operation, including tests of different divertor configurations

- The vacuum vessel volume acts as a high-speed pump to remove neutral gas from the divertor (and diagnostic slot)

Negative flux generates $x$ point on the symmetry axis and diverts outside scrape-off

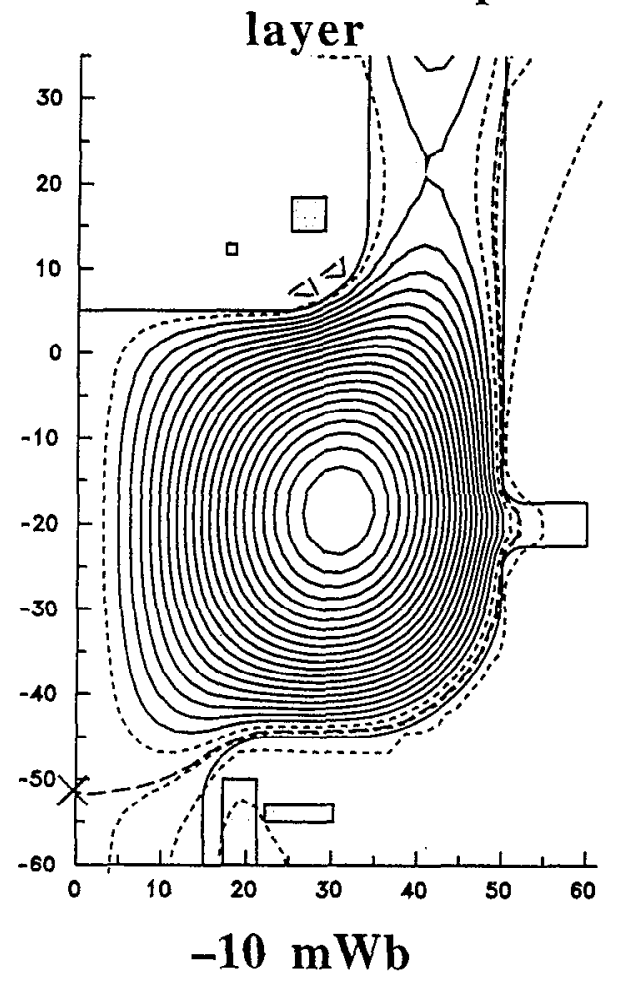

Zero flux (base case) allows access to scrape-off layer or separatrix for a limiter

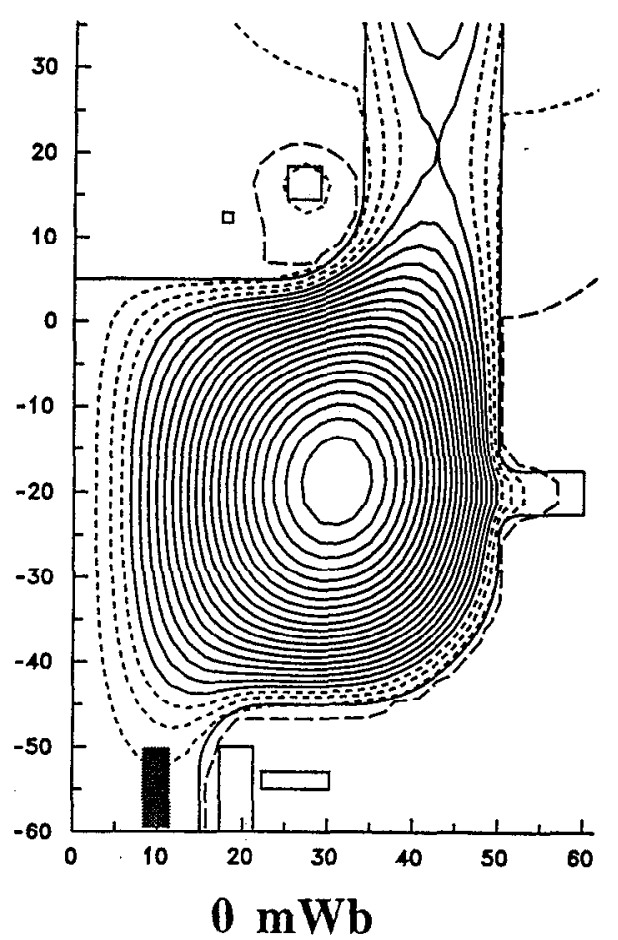

Positive flux diverts gun cathode flux - Operate with anode in divertor (Taylor

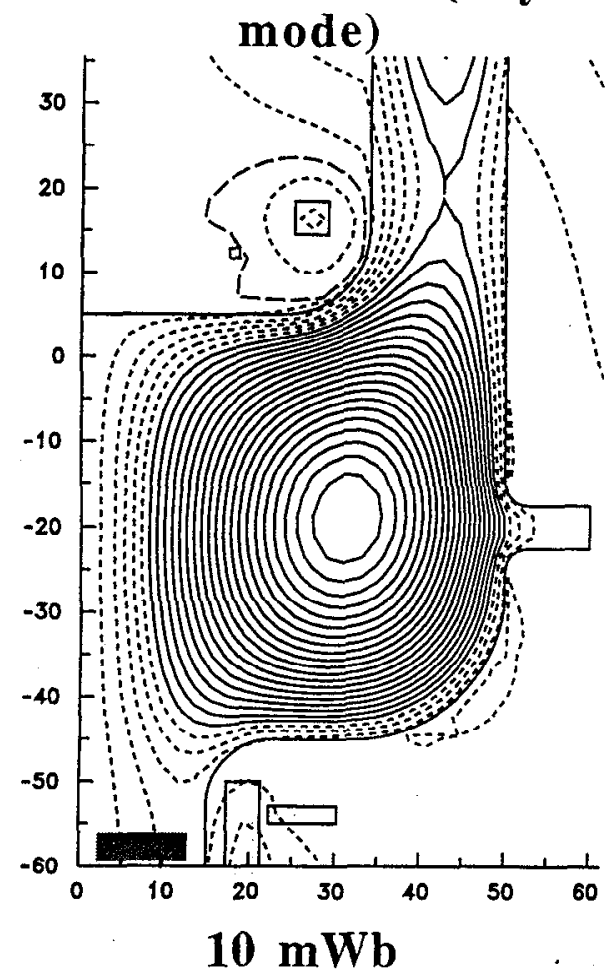

Figure 17. CORSICA calcuated magnetic flux surfaces modeling bias coil control to enable future new SSPX configurations. 


\section{Transport modeling - enhanced buildup losses}

- Calculation using the Fowler model with enhanced losses during buildup:

$$
\chi=\chi_{R-R}\left|1+\frac{\partial / \partial t \int^{V_{\psi}} d V B^{2} / 2 \mu_{0}}{P_{\Omega}}\right|
$$

$\chi_{R-R}$ is the Rechester-Rosenbluth thermal conductivity

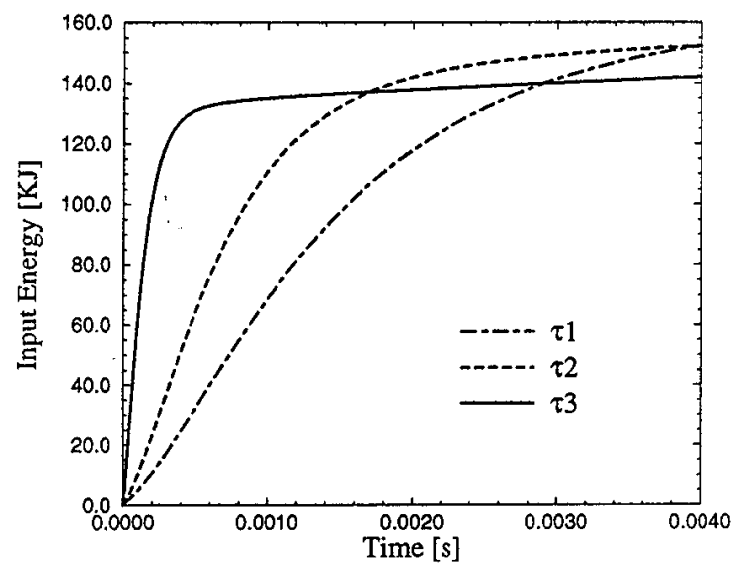

Energy

Input

- The plasma reaches high temperatures

- Earliest for fast buildup times despite the added loss term

- Wall interactions (impurities, etc.) are not included

- Model buildup times (see figures) are $\tau_{1}=1 \mathrm{~ms}, \tau_{2}=0.5 \mathrm{~ms}$, and $\tau_{3}=0.1 \mathrm{~ms}$

Calculations by D. Hua, UC Berkeley

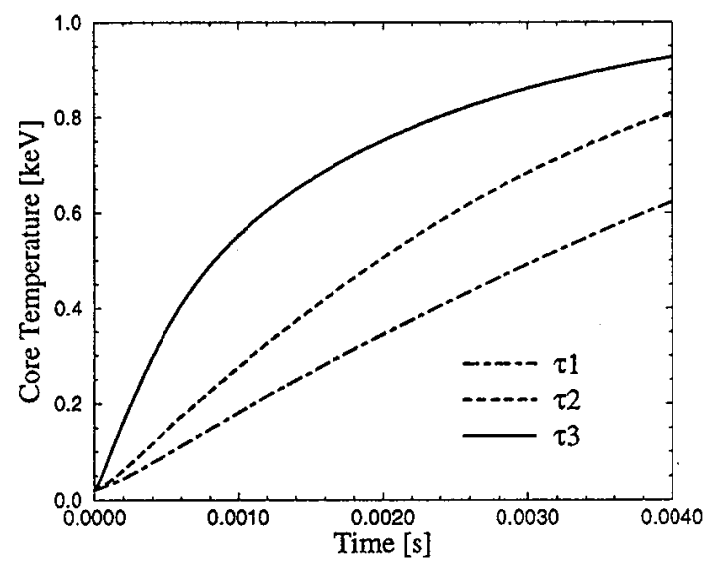

$\mathrm{T}_{\mathrm{e}}$

Figure 18. CORSICA used to model transport in SSPX in order to study plasma energy buildup. 
